\title{
LIFSHITZ TAIL FOR CONTINUOUS ANDERSON MODELS DRIVEN BY LÉVY OPERATORS
}

\author{
KAMIL KALETA AND KATARZYNA PIETRUSKA-PAEUBA
}

\begin{abstract}
We investigate the behavior near zero of the integrated density of states $\ell$ for random Schrödinger operators $\Phi(-\Delta)+V^{\omega}$ in $L^{2}\left(\mathbb{R}^{d}\right), d \geqslant 1$, where $\Phi$ is a complete Bernstein function such that for some $\alpha \in(0,2]$, one has $\Phi(\lambda) \asymp \lambda^{\alpha / 2}, \lambda \searrow 0$, and $V^{\omega}(x)=$ $\sum_{\mathbf{i} \in \mathbb{Z}^{d}} q_{\mathbf{i}}(\omega) W(x-\mathbf{i})$ is a random nonnegative alloy-type potential with compactly supported single site potential $W$. We prove that there are constants $C, \widetilde{C}, D, \widetilde{D}>0$ such that

$$
-C \leqslant \liminf _{\lambda \searrow 0} \frac{\lambda^{d / \alpha}}{\left|\log F_{q}(D \lambda)\right|} \log \ell(\lambda) \quad \text { and } \quad \limsup _{\lambda \searrow 0} \frac{\lambda^{d / \alpha}}{\left|\log F_{q}(\widetilde{D} \lambda)\right|} \log \ell(\lambda) \leqslant-\widetilde{C},
$$

where $F_{q}$ is the common cumulative distribution function of the lattice random variables $q_{\mathrm{i}}$. In particular, we identify how the behavior of $\ell$ at zero depends on the lattice configuration. For typical examples of $F_{q}$ the constants $D$ and $\widetilde{D}$ can be eliminated from the statement above. We combine probabilistic and analytic methods which allow to treat, in a unified manner, both local and non-local kinetic terms such as the Laplace operator, its fractional powers and the quasi-relativistic Hamiltonians.
\end{abstract} $47 \mathrm{G} 30$

MSC Subject Classification (2010): Primary 82B44, 60K37, 60G51; Secondary 47D08,

Keywords: Bernstein functions, Lévy processes, Random local and nonlocal Schrödinger operator, Alloy-type potential, non-local Anderson problem, Integrated density of states, Lifshitz tail, Tauberian theorem

\section{IntroduCtion AND STATEMENT OF RESUlts}

Let $\Phi$ be a complete Bernstein function such that $\lim _{\lambda \searrow_{0}} \Phi(\lambda)=0$ and let

$$
H^{\omega}=\Phi(-\Delta)+V^{\omega}
$$

be a random Schrödinger operator in $L^{2}\left(\mathbb{R}^{d}\right)$ with an alloy-type potential

$$
V^{\omega}(x)=\sum_{\mathbf{i} \in \mathbb{Z}^{d}} q_{\mathbf{i}}(\omega) W(x-\mathbf{i}), \quad x \in \mathbb{R}^{d},
$$

where $\left\{q_{\mathbf{i}}\right\}_{\mathbf{i} \in \mathbb{Z}^{d}}$ is a sequence of i.i.d. nonnegative and nondegenerate random variables over the probability space $(\Omega, \mathcal{A}, \mathbb{Q})$, with cumulative distribution function $F_{q}(t)=\mathbb{Q}[q \leqslant t]$, and $W: \mathbb{R}^{d} \rightarrow[0, \infty)$ is a sufficiently regular nonnegative single-site potential. The class of kinetic terms $\Phi(-\Delta)$ we consider contains both local operators such as the classical Laplacian $-\Delta$ (for $\Phi(\lambda)=\lambda$ ) as well as a wide range of non-local pseudo-differential operators which are of great interest in mathematical physics. The most prominent examples in this class are the fractional Laplacians $(-\Delta)^{\alpha / 2}$ (for $\Phi(\lambda)=\lambda^{\alpha / 2}, \alpha \in(0,2)$ ) and the quasi-relativistic operators $\left(-\Delta+m^{2 / \vartheta}\right)^{\vartheta / 2}-m\left(\right.$ for $\left.\Phi(\lambda)=\left(\lambda+m^{2 / \vartheta}\right)^{\vartheta / 2}-m, \vartheta \in(0,2), m>0\right)$ [5, 14, 35].

Under the regularity assumptions (B), (Q) and (W) (stated below) on the Bernstein function $\Phi$, the distribution function $F_{q}$, and the single-site potential $W$, respectively, we study the asymptotic behavior of the integrated density of states (IDS) for the operator $H^{\omega}$ at the bottom of its spectrum. The precise definition of IDS is given in Section 2.6. We will use the same letter $\ell$ to denote both the IDS (a measure) and its cumulative distribution function, i.e. $\ell(\lambda):=\ell([0, \lambda])$.

Research supported by the National Science Center, Poland, grant no. 2015/17/B/ST1/01233. 
In 1965, I.M. Lifshitz discovered, on physical grounds, that the density of states $\ell(\lambda)$ of certain random Hamiltonian $H^{\omega}=H_{0}+V^{\omega}$ displays unusually fast decay near the bottom $\lambda_{0}$ of its spectrum $\sigma\left(H^{\omega}\right)$ : it behaves roughly as $\exp \left(-c\left(\lambda-\lambda_{0}\right)^{-d / 2}\right)$ [25. Since then, such a behavior has been called 'the Lifshitz tail'. For $H_{0}=-\Delta$ (or $-\Delta$ with periodic potential) and for various classes of random potentials $V^{\omega}$ it has been widely studied and rigourously proven in both continuous (Benderskii and Pastur [1, Friedberg and Luttinger [10, Luttinger [26], Nakao [29], Pastur [30], Kirsch and Martinelli [19, 20], Mezincescu [27, Kirsch and Simon [21], Kirsch and Veselić [22]) and discrete (Fukushima [11], Fukushima, Nagai and Nakao [12], Nagai [28], Romerio and Wreszinski [34], Simon [37]) settings (both of these lists are far from being complete). Note in passing that these random Hamiltonians typically exhibit the so-called spectral localization (see e.g. Combes and Hislop [8], Bourgain and Kenig [3],Germinet, Hislop and Klein [13], and the references in these papers). It is known that the Lifshitz singularity is a strong indication for this property to hold and rigorous proofs of localization often use the approximation of the IDS resulting from the Lifshitz asymptotics (see e.g. the discussion in the papers by Klopp [23, 24] and Kirsch and Veselić [22]).

One of the best studied cases in the classical setting are the Poisson-type potentials. These random potentials, defined as

$$
V^{\omega}(x)=\int_{\mathbb{R}^{d}} W(x-y) \mu^{\omega}(\mathrm{d} y),
$$

where $W$ is a sufficiently regular non-negative profile function and $\mu^{\omega}$ is the Poisson random measure on $\mathbb{R}^{d}$ with intensity $\nu>0$, were first rigorously investigated in the papers of Nakao [29] and Pastur [30]. This special framework allowed to apply the Donsker-Varadhan large deviations technique to show the following strong statement:

$$
\lim _{\lambda \searrow 0} \lambda^{d / 2} \log \ell(\lambda)=-C(d) \nu
$$

where $C(d)$ is an explicit constant. Later, this result was extended by Okura [32] to more general operators $H_{0}=-L$, where $L$ is a pseudo-differential operator with sufficiently regular Fourier symbol $\Psi(\xi)$ (this class includes $\Delta$ and many other non-local operators $-\Phi(-\Delta)$ studied in the present paper), with Poissonian random potential $V^{\omega}$. It was also the subject of research on less regular spaces such as fractals, see [31, 16].

Back to the lattice (alloy-type) potentials, let us emphasize that the Lifshitz behavior for $H_{0}=-\Delta$ and non-negative potentials as in (1.2) (with $\lambda_{0}=0$ ) was typically established in a form somewhat weaker than (1.3), namely

$$
\lim _{\lambda \searrow 0} \frac{\log |\log \ell(\lambda)|}{\log \lambda}=-\kappa
$$

Below, this will be referred to as the ' $\log \log$ statement'. Here $\kappa=d / 2$ or $\kappa=d / \beta$, where $\beta>0$ is the parameter describing the decay rate of the single site potential $W$ at infinity (see e.g. Kirsch and Martinelli [20, Theorem 7], Kirsch and Simon [21, Theorem 1]). Kirsch and Martinelli also gave some sufficient conditions for the existence of constants $C, \widetilde{C}>0$ such that $e^{-C \lambda^{-d / 2}} \leqslant \ell(\lambda) \leqslant e^{-\widetilde{C} \lambda^{-d / 2}}$, for $\lambda$ close to zero.

Recently, Kaleta and Pietruska-Pałuba [17] considered the case of $H_{0}=(-\Delta)^{\alpha / 2}, \alpha \in(0,2]$, and alloy-type potentials $V^{\omega}$ as in (1.2) with bounded, compactly supported single-site potentials that are separated from zero in a vicinity of zero, under the assumption that $F_{q}(\kappa)>0$ for $\kappa>0$. The authors were able to prove that

$$
\lim _{\lambda \searrow 0} \lambda^{d / \alpha} \log \ell(\lambda)=-C(d, \alpha) \log \frac{1}{F_{q}(0)} .
$$

Clearly, this limit is finite if and only if $F_{q}(0)>0$, i.e. when the distribution of the lattice random variables has an atom at zero. If this holds, the resulting asymptotics is very close to that in 
(1.3), known for the Poissonian model (see the more detailed discussion in the introduction of the cited paper). This result shows also that when $F_{q}(0)=0$, then the limit is infinite, so $\lambda^{d / \alpha}$ is not a correct normalization term for (1.5). The correct rate for $\ell(\lambda)$ should be faster and depend on the behavior of the distribution of the lattice random variables near zero.

This feature motivated our research in the present paper. Our main result, addressing this problem, is given in Theorem 1.1 below. Interestingly, quite often, such a delicate influence is lost by taking the double logarithm in the 'loglog statements' as in (1.4) (cf. Example1.3(1)-(2) below). As we will see below, a more detailed analysis is required in this case.

Before we pass to the presentation of our results, we introduce the framework assumptions. They read as follows.

(B) $\Phi$ is a complete Bernstein function satisfying

$$
\lim _{\lambda \rightarrow \infty} \frac{\Phi(\lambda)}{\log \lambda}=\infty
$$

and such that there there exist $\alpha \in(0,2], C_{1}, C_{2}, \lambda_{0}>0$ for which

$$
C_{1} \lambda^{\alpha / 2} \leqslant \Phi(\lambda) \leqslant C_{2} \lambda^{\alpha / 2}, \quad \lambda<\lambda_{0} .
$$

(Q) The random variables $q_{\mathbf{i}}, \mathbf{i} \in \mathbb{Z}^{d}$, defined on a probability space $(\Omega, \mathcal{A}, \mathbb{Q})$, are independent copies of a non-negative and non-degenerate (i.e. not equal to a constant a.s.) random variable $q$. Moreover, denoting by $F_{q}$ the cumulative distribution function of $q$, i.e. $F_{q}(\kappa):=\mathbb{Q}(q \leqslant \kappa)$, we assume that $F_{q}(\kappa)>0$ for all $\kappa>0$ and that there exists $\kappa_{0}>0$ such that $\left.F_{q}\right|_{\left[0, \kappa_{0}\right]}$ is continuous (left discontinuity at 0 is permitted).

(W) The single-site potential $W: \mathbb{R}^{d} \rightarrow \mathbb{R}_{+}$has compact support included in $\left[-M_{0}, M_{0}\right]^{d}$, for certain $M_{0} \in \mathbb{Z}_{+}, W \in L^{2}\left(\mathbb{R}^{d}\right)$, and $\|W\|_{2}>0$. Moreover, $W$ belongs to the Kato class of the operator $\Phi(-\Delta)$.

For precise definitions of Bernstein functions, Kato class etc. we refer the reader to Section 2 below. Note that our assumption (B) covers a wide range of complete Bernstein functions $\Phi$ leading to various important classes of operators $\Phi(-\Delta)$. Some of them are listed in Example 2.2 in Section 2.2. For instance, if $\Phi(\lambda)=\lambda^{\alpha / 2}, \alpha \in(0,2]$ (the Laplace operator and its fractional powers), then (1.7) clearly holds with the same $\alpha$. For $\Phi(\lambda)=\left(\lambda+m^{2 / \vartheta}\right)^{\vartheta / 2}-m$, with $\vartheta \in(0,2)$ and $m>0$ (the quasi-relativistic operators), we have to choose $\alpha=2$ in (1.7).

The assumption $(\mathbf{W})$ is also quite general. It automatically holds for nonnegative $W$ 's that are bounded and of compact support. However, singular functions $W$ are allowed as well (further details are given in Example 2.6] in Section 2.4).

Our main result reads as follows.

Theorem 1.1. Let the assumptions (B), (Q) and (W) hold and let $\ell$ be the IDS of the random Schrödinger operator defined in (1.1)-(1.2). Then there exist constants $C, \widetilde{C}, D, \widetilde{D}>0$ such that

$$
-C \leqslant \liminf _{\lambda \searrow 0} \frac{\lambda^{d / \alpha}}{g(D / \lambda)} \log \ell(\lambda)
$$

and

$$
\limsup _{\lambda \searrow 0} \frac{\lambda^{d / \alpha}}{g(\widetilde{D} / \lambda)} \log \ell(\lambda) \leqslant-\widetilde{C},
$$

where $g(x)=\log \frac{1}{F_{q}\left(D_{0} / x\right)}$ and $D_{0}$ is another nonnegative constant (defined in (3.1)).

Interestingly, the rate for $\log \ell(\lambda)$ can be fully factorized: it depends on the kinetic term $\Phi(-\Delta)$ through $\lambda^{d / \alpha}$ and, separately, on the potential $V^{\omega}$ - through the function $g(x)$ describing the common distribution of lattice random variables. To the best of our knowledge, such a general description of the behavior of the IDS at the bottom of the spectrum, involving the dependence of the lattice configuration, was not known before. This is illustrated by Example 1.3 below. 
Moreover, for functions $g$ with sufficiently regular growth at infinity (see Example 1.3 below), the constants $D, \widetilde{D}$ in Theorem 1.1 can be eliminated and we arrive at a single statement:

$$
-C \leqslant \liminf _{\lambda \searrow 0} \frac{\lambda^{d / \alpha}}{\log \frac{1}{F_{q}(\lambda)}} \log \ell(\lambda) \leqslant \limsup _{\lambda \searrow 0} \frac{\lambda^{d / \alpha}}{\log \frac{1}{F_{q}(\lambda)}} \log \ell(\lambda) \leqslant-\widetilde{C} .
$$

In particular, if the distribution of the lattice random variables has an atom at zero, i.e. $F_{q}(0)>0$, then the statement simplifies to

$$
-C \log \frac{1}{F_{q}(0)} \leqslant \liminf _{\lambda \searrow 0} \lambda^{d / \alpha} \log \ell(\lambda) \leqslant \limsup _{\lambda \searrow 0} \lambda^{d / \alpha} \log \ell(\lambda) \leqslant-\widetilde{C} \log \frac{1}{F_{q}(0)},
$$

i.e. we obtain the rate known from the continuous Poisson model (cf. [32]).

It is useful to give the following interpretation of our main result (cf. [38, Remark 3.6(1)] and the comments following [17, Theorem 1.1]).

Remark 1.2. (Interpretation) Suppose $B\left(r_{\lambda}\right)$ is a ball with radius $r_{\lambda} \asymp \lambda^{-1 / \alpha}$. Clearly, $\left|B\left(r_{\lambda}\right)\right| \asymp \lambda^{-d / \alpha}$, and due to the condition (1.7) and [7, Theorem 4.4] the principal Dirichlet eigenvalue of the operator $\Phi(-\Delta)$ constrained to $B\left(r_{\lambda}\right)$ is proportional to $\lambda$ as $\lambda \searrow 0$. Then, assuming that $D=\widetilde{D}=1$ (this is often the case - see Example 1.3 below) our main result from Theorem 1.1 can be written as

$$
F_{q}\left(D_{0} \lambda\right)^{C\left|B\left(r_{\lambda}\right)\right|} \leqslant \ell(\lambda) \leqslant F_{q}\left(D_{0} \lambda\right)^{\widetilde{C}\left|B\left(r_{\lambda}\right)\right|}, \text { for } \lambda \searrow 0 .
$$

Since $F_{q}\left(D_{0} \lambda\right)$ is the probability of the $q_{\mathbf{i}}$ being not larger than $D_{0} \lambda$ at any given lattice point, $\ell(\lambda)$ behaves roughly as the probability that in the ball with ground state eigenvalue comparable to $\lambda$, all random variables $q_{\mathrm{i}}$ 's are smaller than $D_{0} \lambda$.

A direct consequence of Theorem 1.1 is the following 'loglog statement' which generalizes (1.4) (see Corollary (5.4): if $\lim _{x \rightarrow \infty} \frac{\log g(x)}{\log x}$ exists, then

$$
\lim _{\lambda \searrow 0} \frac{\log |\log \ell(\lambda)|}{\log \lambda}=-\frac{d}{\alpha}-\lim _{x \rightarrow \infty} \frac{\log g(x)}{\log x} .
$$

Our main result is illustrated by four different examples of distribution functions $F_{q}$, yielding distinct asymptotics of the IDS near zero (more detailed discussion of these examples can be found in Section (6). Roughly speaking, the faster the decay of $F_{q}$ at zero, the faster the decay of $\ell(\lambda)$ at zero as well.

Example 1.3. Suppose that the assumptions (B) and (W) hold. Then there exist constants $C, \widetilde{C}>0$ such that:

(1) atom at zero: if there exists $\kappa_{0}>0$ such that $F_{q}$ is continuous on $\left[0, \kappa_{0}\right]$ and $F_{q}(0)>0$, then

$-C \leqslant \liminf _{\lambda \searrow 0} \lambda^{d / \alpha} \log \ell(\lambda) \leqslant \limsup _{\lambda \searrow 0} \lambda^{d / \alpha} \log \ell(\lambda) \leqslant-\widetilde{C} \quad$ and $\quad \lim _{\lambda \searrow 0} \frac{\log |\log \ell(\lambda)|}{\log \lambda}=-\frac{d}{\alpha}$.

(2) polynomial decay at zero: if there exists $\kappa_{0}>0$ such that $F_{q}$ is continuous on $\left[0, \kappa_{0}\right]$ and $c_{1} \kappa^{\gamma_{1}} \leqslant F_{q}(\kappa) \leqslant c_{2} \kappa^{\gamma_{2}}, \kappa \in\left[0, \kappa_{0}\right]$, for some $\gamma_{1}, \gamma_{2}, c_{1}, c_{2}>0$, then

$-C \leqslant \liminf _{\lambda \searrow 0} \frac{\lambda^{d / \alpha}}{\log \lambda} \log \ell(\lambda) \leqslant \limsup _{\lambda \searrow 0} \frac{\lambda^{d / \alpha}}{\log \lambda} \log \ell(\lambda) \leqslant-\widetilde{C} \quad$ and $\quad \lim _{\lambda \searrow 0} \frac{\log |\log \ell(\lambda)|}{\log \lambda}=-\frac{d}{\alpha}$.

(3) exponential decay at zero: if $F_{q}(\kappa)=\mathrm{e}^{-\frac{1}{\kappa^{\gamma}}}, \kappa>0$, for some $\gamma>0$, then

$-C \leqslant \liminf _{\lambda \searrow 0} \lambda^{\frac{d}{\alpha}+\gamma} \log \ell(\lambda) \leqslant \limsup _{\lambda \searrow 0} \lambda^{\frac{d}{\alpha}+\gamma} \log \ell(\lambda) \leqslant-\widetilde{C} \quad$ and $\quad \lim _{\lambda \searrow 0} \frac{\log |\log \ell(\lambda)|}{\log \lambda}=-\frac{d}{\alpha}-\gamma$. 
(4) double-exponential decay at zero: if $F_{q}(\kappa)=\mathrm{e}^{1-\mathrm{e}^{\frac{1}{\kappa}}}, \kappa>0$, then there exist constants $D_{1}, D_{2}>0$ such that

$$
-C \leqslant \liminf _{\lambda \searrow 0} \lambda^{d / \alpha} \mathrm{e}^{-\frac{D_{1}}{\lambda}} \log \ell(\lambda) \quad \text { and } \quad \limsup _{\lambda \searrow 0} \lambda^{d / \alpha} \mathrm{e}^{\frac{-D_{2}}{\lambda}} \log \ell(\lambda) \leqslant-\widetilde{C}
$$

and

$$
C \leqslant \liminf _{\lambda \searrow 0} \lambda \log |\log \ell(\lambda)| \leqslant \limsup _{\lambda \searrow 0} \lambda \log |\log \ell(\lambda)| \leqslant \widetilde{C} .
$$

We call this behavior the super-Lifchitz tail.

As Example 1.3 (3)-(4) above indicates, the contribution coming from the lattice configuration might not be just the correction term (i.e. a lower order term). Actually, its order may be polynomial or even faster, so this term may be the leading one. Such an effect was not observed before. Note also that the distribution functions $F_{q}$ as in Example 1.3 (1)-(2) satisfy the assumptions of the paper by Kirsch and Simon [21, Theorem 1], who established the Lifshitz singularity in the 'loglog' form (1.4) for the random Schrödinger operators based on Laplacian (i.e. for $\Phi(\lambda)=\lambda$ ). For the case (1), a version of this results was first obtained by Kirsch and Martinelli [20, Theorem 7]. Our present work extends and improves these results to the case of compactly supported single-site potentials (see Section 6.2 for a broader discussion).

Our approach in the present paper is based on a combination of analytic and probabilistic methods. To make the paper easier accessible to the analytic community, in Section 2 we have included a detailed description of subordinate processes and their evolution semigroups, Kato classes, Schrödinger operators, Feynman-Kac formula etc. The reader familiar with those topics may just give a cursory look at this section and start the lecture from Section 3 , maybe coming back to the previous section for the notation.

Our argument is constructed as follows. We first find proper estimates for the Laplace transform $L(t)$ of the IDS (Sections 3, 4), and then we transform them to the bounds on the IDS itself (Section 5). The proof of the upper bound for $L(t)$ (Theorem 3.1) is the most demanding part of this work. It consists of several steps which are presented in Sections 3.2, 3.3 and 3.4, respectively. In the first step, using the stochastic Feynman-Kac representation and monotonicity, we estimate $L(t)$ by the trace of the evolution semigroup of the Schrödinger operator $\Phi\left(\Delta_{M}\right)+V_{M}^{\omega}$, where $\Delta_{M}$ is the Laplace operator on a torus of given size $M \geqslant M_{0}$, and $V_{M}^{\omega}$ is an alloy-type potential defined for the periodized lattice configuration. With this preparation, in the next step, we are able to apply in our framework a beautiful idea we learned from the papers by Simon [37] and Kirsch and Simon [21], which is based on an application of the Temple's inequality (Proposition 3.2). In [21, Proposition 3] the authors proved that if the ground state eigenvalue of a random Schrödinger operator constrained to a box of size $L$ (with Neumann boundary conditions) is smaller than a given number $\lambda>0$, then the number of those lattice random variables in this box which are less than $4 \lambda$ is larger than $L^{d} / 2$. In general, our approach in the present paper is different from that in the cited papers (we estimate the Laplace transform directly and we do not employ the Dirichlet-Neumann bracketing), but we came up with a version of this implication suitable for our setting (Lemma 3.4). With a given control level $\delta>0$ and fixed $M$, we divide all lattice configurations into two disjoint subsets: $\mathcal{A}_{M, \delta}$ and $\mathcal{A}_{M, \delta}^{c}$. In the first set, there is a lot of built-in randomness, which permits to find a proper lower-scaling bound for the ground state eigenvalue of the operator $\Phi\left(\Delta_{M}\right)+V_{M}^{\omega}$ with $\omega \in \mathcal{A}_{M, \delta}$. The probability of $\mathcal{A}_{M, \delta}^{c}$ is estimated by means of a Bernstein-type estimate for the binomial distribution (Lemma 3.5). We then need to balance the two - by optimization we find $M=M(t)$ for which both summands are of the same order. This leads us directly to the identification of the correct rate function for $\log L(t)$.

The proof of the lower bound for the Laplace transform $L(t)$ (Theorem 4.2 of Section 4) is more direct. We restrict the integration to the set of special lattice configurations for which 
we can reduce the problem to a careful analysis of the evolution semigroups associated to nonrandom Schrödinger operators $\Phi(-\Delta)+\frac{C}{M^{\alpha}} \sum_{\mathbf{i} \in[-M, 2 M)^{d}} W(x-\mathbf{i})$ constrained to the box of size $M$ (with Dirichlet conditions).

As the last step, in Section 5 we transform the statements concerning the asymptotical behavior of $L(t)$ at infinity into statements for $\ell(\lambda)$ near zero. This is done by an application of a Tauberian-type theorem. Let us emphasize that the the asymptotic rates for $\log L(t)$ identified in our Theorems 3.1 and 4.2 are typically more complicated than $t^{\gamma}, \gamma \in(0,1)$ (which are the rates e.g. in the Poissonian case), as they may comprise also lower order terms (see the more detailed discussion of the specific cases in Section [6). This causes additional difficulties as the Tauberian theorems available in the literature did not cover such a case. Therefore, we had to prove a more general Tauberian theorem which is specialized to work in our present framework (Theorem 5.1).

Convention concerning constants. There are four structural constants of this paper $-C_{1}, C_{2}$ of Assumption (B), $M_{0}$ of assumption (W), and $D_{0}$ of formula (3.1). Their values are kept fixed throughout the paper. The value of other roman-type constants (both lower- and upper-case) is not relevant and can change at each appearance. When we need to keep track of the dependence between technical constants, we number them inside the proofs consecutively as $c_{1}, c_{2}, \ldots$

\section{Bernstein FUnCTIONS AND CORRESPONDING SCHRÖDINGER OPERATORS}

As indicated in the Introduction, the approach of this paper is based on a combination of probabilistic and analytic methods. The Schrödinger semigroups we consider are represented by the Feynman-Kac formula with respect to Lévy processes that are obtained via subordination (random time change) of the standard Brownian motion in $\mathbb{R}^{d}$. We start our preparation by giving the necessary preliminaries on Bernstein functions, related stochastic processes, and unbounded operators, then we discuss the class of random potentials studied in this paper. Finally, we introduce the corresponding Schrödinger operators and discuss their properties.

2.1. Bernstein functions and subordinators. A function $\Phi:(0, \infty) \rightarrow[0, \infty)$ is called completely monotone if it is smooth and satisfies $(-1)^{n} \Phi^{(n)}(x) \geqslant 0$, for every $x>0$ and $n \in \mathbb{Z}_{+}$. We call $\Phi$ a Bernstein function if it is a nonnegative and smooth function with completely monotone derivative. Our standard reference to Bernstein functions, corresponding operators, and stochastic processes is the monograph [35].

It is known that every Bernstein function $\Phi$ admits the representation

$$
\Phi(\lambda)=a+b \lambda+\int_{(0, \infty)}\left(1-\mathrm{e}^{-\lambda u}\right) \rho(\mathrm{d} u)
$$

where $a, b \geqslant 0$ and $\rho$ is a Lévy measure, i.e. a nonnegative Radon measure on $(0, \infty)$ such that $\int_{(0, \infty)}(u \wedge 1) \rho(\mathrm{d} u)<\infty$. A Bernstein function is said to be a complete Bernstein function if its Lévy measure has a completely monotone density with respect to the Lebesgue measure.

Bernstein functions $\Phi$ with $\lim _{\lambda \searrow 0} \Phi(\lambda)=0$ (i.e. $a=0$ ) are in one-to-one correspondence with subordinators. The stochastic process $S=\left(S_{t}\right)_{t \geqslant 0}$ on a probability space $\left(\Omega_{0}, \mathcal{F}, \mathcal{P}\right)$ is called a subordinator if it is a nondecreasing Lévy process in $\mathbb{R}_{+}$, i.e. a process with càdlàg paths (right continuous with left limits finite) starting from 0 , with stationary and independent increments. The laws of $S$, given by $\eta_{t}(\mathrm{~d} u):=\mathcal{P}\left(S_{t} \in \mathrm{d} u\right), t \geqslant 0$, form a convolution semigroup of probability measures on $[0, \infty)$ which is uniquely determined by the Laplace transform

$$
\int_{[0, \infty)} \mathrm{e}^{-\lambda u} \eta_{t}(\mathrm{~d} u)=\mathrm{e}^{-t \Phi(\lambda)}, \quad \lambda>0
$$


where the Laplace exponent $\Phi$ is a Bernstein function such that $\lim _{\lambda \searrow_{0}} \Phi(\lambda)=0$. The number $b$ and the measure $\rho$ are called the drift term and the Lévy measure of the subordinator $S$, respectively.

Under (1.6) we have $\lim _{\lambda \rightarrow \infty} \Phi(\lambda)=\infty$, and therefore either $b>0$ or $\int_{(0, \infty)} \rho(\mathrm{d} u)=\infty$. We also easily see from (2.2) that in this case $\eta_{t}(\{0\})=0$, for every $t>0$. The following lemma will be an important tool below. It is based on standard calculations, but we include here a short proof for the reader's convenience.

Lemma 2.1. For every $\gamma>0$ there is a constant $C=C(\gamma)$ such that

$$
\int_{[0, \infty)} u^{-\gamma} \eta_{t}(\mathrm{~d} u)=C \int_{0}^{\infty} \mathrm{e}^{-t \Phi\left(\lambda^{1 / \gamma}\right)} \mathrm{d} \lambda, \quad t>0 .
$$

Under the assumption (B), for every $t_{0}>0$ there exists a constant $\widetilde{C}=\widetilde{C}\left(t_{0}\right)$ such that

$$
\int_{(0, \infty)} u^{-\gamma} \eta_{t}(\mathrm{~d} u) \leqslant \widetilde{C} t^{-2 \gamma / \alpha}, \quad t \geqslant t_{0}
$$

In particular, for every $t_{0}>0$,

$$
\sup _{t \geqslant t_{0}} \int_{(0, \infty)} u^{-\gamma} \eta_{t}(\mathrm{~d} u)<\infty
$$

Proof. First note that by (2.2) we have $\mathrm{e}^{-t \Phi\left(\lambda^{1 / \gamma}\right)}=\int_{0}^{\infty} \mathrm{e}^{-\left(\lambda u^{\gamma}\right)^{1 / \gamma}} \eta_{t}(\mathrm{~d} u), \lambda, t>0$. Then, by Fubini-Tonelli and the substitution $\vartheta=u^{\gamma} \lambda$, we get

$$
\int_{0}^{\infty} \mathrm{e}^{-t \Phi\left(\lambda^{1 / \gamma}\right)} \mathrm{d} \lambda=\int_{0}^{\infty} \int_{0}^{\infty} \mathrm{e}^{-\left(\lambda u^{\gamma}\right)^{1 / \gamma}} \mathrm{d} \lambda \eta_{t}(\mathrm{~d} u)=\int_{0}^{\infty} \mathrm{e}^{-\vartheta^{1 / \gamma}} d \vartheta \int_{0}^{\infty} u^{-\gamma} \eta_{t}(\mathrm{~d} u),
$$

which gives the first equality with $C=\left(\int_{0}^{\infty} \mathrm{e}^{-\vartheta^{1 / \gamma}} \mathrm{d} \vartheta\right)^{-1}$. Moreover, for every fixed $t_{0}>0$,

$$
\sup _{t \geqslant t_{0}} \int_{0}^{\infty} u^{-\gamma} \eta_{t}(\mathrm{~d} u)=C \sup _{t \geqslant t_{0}} \int_{0}^{\infty} \mathrm{e}^{-t \Phi\left(\lambda^{1 / \gamma}\right)} \mathrm{d} \lambda \leqslant C \int_{0}^{\infty} \mathrm{e}^{-t_{0} \Phi\left(\lambda^{1 / \gamma}\right)} \mathrm{d} \lambda .
$$

Assume now (B) and fix $t_{0}>0$. Observe that by (1.6) there exists $\widetilde{\lambda}_{0}>\lambda_{0}$ such that $t_{0} \Phi\left(\lambda^{1 / \gamma}\right) \geqslant 2 \log \lambda$, for $\lambda \geqslant \widetilde{\lambda}_{0}$. Also, by decreasing the constant $C_{1}>0$ if needed, we may assume that the lower bound in (1.7) holds with $\lambda_{0}$ replaced with $\widetilde{\lambda}_{0}$ (this is possible due to monotonicity and strict positivity of $\Phi$ on $(0, \infty))$. With this in mind,

$$
\begin{aligned}
\int_{0}^{\infty} u^{-\gamma} \eta_{t}(\mathrm{~d} u)=C \int_{0}^{\infty} \mathrm{e}^{-t \Phi\left(\lambda^{1 / \gamma}\right)} \mathrm{d} \lambda & \leqslant C\left(\int_{0}^{\widetilde{\lambda}_{0}} \mathrm{e}^{-t \Phi\left(\lambda^{1 / \gamma}\right)} \mathrm{d} \lambda+\mathrm{e}^{-\left(t-t_{0}\right) \Phi\left(\widetilde{\lambda}_{0}^{1 / \gamma}\right)} \int_{\widetilde{\lambda}_{0}}^{\infty} \mathrm{e}^{-t_{0} \Phi\left(\lambda^{1 / \gamma}\right)} \mathrm{d} \lambda\right) \\
& \leqslant C\left(\int_{0}^{\widetilde{\lambda}_{0}} \mathrm{e}^{-t A_{1} \lambda^{\alpha /(2 \gamma)}} \mathrm{d} \lambda+\mathrm{e}^{-\left(t-t_{0}\right) \Phi\left(\widetilde{\lambda}_{0}^{1 / \gamma}\right)} \int_{\widetilde{\lambda}_{0}}^{\infty} \mathrm{e}^{-2 \log \lambda} \mathrm{d} \lambda\right) .
\end{aligned}
$$

Using the substitution $\vartheta=t^{2 \gamma / \alpha} \lambda$ for the first integral and the fact that there exists a constant $c=c\left(t_{0}\right)>0$ such that $e^{-\left(t-t_{0}\right) \Phi\left(\widetilde{\lambda}_{0}^{1 / \gamma}\right)} \leqslant c t^{-2 \gamma / \alpha}$, for $t \geqslant t_{0}$, we finally get

$$
\int_{0}^{\infty} u^{-\gamma} \eta_{t}(\mathrm{~d} u) \leqslant C\left(\int_{0}^{\infty} \mathrm{e}^{-A_{1} \vartheta^{\alpha /(2 \gamma)}} \mathrm{d} \vartheta+\frac{c}{\widetilde{\lambda}_{0}}\right) t^{-2 \gamma / \alpha}=\widetilde{C} t^{-2 \gamma / \alpha}
$$

This completes the proof.

2.2. Operators $\Phi(-\Delta)$ and subordinate Brownian motions. Denote by $\left\{G_{t}: t \geqslant 0\right\}$ the classical heat semigroup acting on $L^{2}\left(\mathbb{R}^{d}\right)$, i.e.

$$
G_{t} f(x)=\int_{\mathbb{R}^{d}} g_{t}(x-y) f(y) \mathrm{d} y, \quad f \in L^{2}\left(\mathbb{R}^{d}\right), t>0,
$$

where $g_{t}(x)=(4 \pi t)^{-d / 2} e^{-|x|^{2} / 4 t}$ is the Gauss-Weierstrass kernel. We have $G_{t}=e^{t \Delta}$, where $\Delta$ is the classical Laplace operator. Recall that it is an unbounded, non-positive definite, self-adjoint 
operator in $L^{2}\left(\mathbb{R}^{d}\right)$. On the probabilistic side, $\left\{G_{t}: t \geqslant 0\right\}$ serves as the transition semigroup of the standard Brownian motion $Z=\left(Z_{t}\right)_{t \geqslant 0}$ in $\mathbb{R}^{d}$, running at twice the usual speed.

Suppose now that $\Phi$ is a Bernstein function such that $\lim _{\lambda \searrow_{0}} \Phi(\lambda)=0$ and let $\left\{\eta_{t}: t \geqslant 0\right\}$ be the convolution semigroup of measures determined by (2.2). With this, we can define

$$
P_{t} f(x):=\int_{[0, \infty)} G_{u} f(x) \eta_{t}(\mathrm{~d} u), \quad f \in L^{2}\left(\mathbb{R}^{d}\right), t \geqslant 0 .
$$

One can check that $P_{t}$ form a strongly continuous semigroup of bounded self-adjoint operators in $L^{2}\left(\mathbb{R}^{d}\right)$ which is referred to as the subordinate heat semigroup; under the assumption (1.6)) (giving $\left.\eta_{t}(\{0\})=0, t>0\right)$ all the $P_{t}^{\prime}$ 's, $t>0$, are integral operators with kernels $p_{t}(x, y):=p_{t}(y-x)$, where

$$
p_{t}(x)=\int_{0}^{\infty} g_{u}(x) \eta_{t}(\mathrm{~d} u), \quad t>0 .
$$

Under the full assumption (B), by Lemma 2.1, we obtain that for every $t_{0}>0$ there exists $C=C\left(t_{0}\right)$ such that

$$
p_{t}(x) \leqslant(4 \pi)^{-d / 2} \int_{0}^{\infty} u^{-d / 2} \eta_{t}(\mathrm{~d} u) \leqslant C t^{-d / \alpha}, \quad x \in \mathbb{R}^{d}, \quad t \geqslant t_{0} .
$$

It is known that $P_{t}=\mathrm{e}^{-t \Phi(-\Delta)}$, where $\Phi(-\Delta)$ is the Fourier multiplier with symbol $\Phi\left(|\xi|^{2}\right)$, i.e. the operator

with the domain

$$
\Phi(-\Delta) f=\mathcal{F}^{-1}\left(\Phi\left(|\cdot|^{2}\right) \mathcal{F} f(\cdot)\right), \quad f \in \mathcal{D}(\Phi(-\Delta))
$$

$$
\mathcal{D}(\Phi(-\Delta))=\left\{f \in L^{2}\left(\mathbb{R}^{d}\right): \Phi\left(|\xi|^{2}\right) \mathcal{F} f(\xi) \in L^{2}\left(\mathbb{R}^{d}\right)\right\}
$$

$\Phi(-\Delta)$ is an unbounded, non-negative definite, self-adjoint operator on $L^{2}\left(\mathbb{R}^{d}\right)$ and $\mathcal{D}(\Phi(-\Delta))$ contains $\mathcal{D}(-\Delta)$. The quadratic form associated with this operator is given by

$$
\mathcal{E}(f, f)=\int_{\mathbb{R}^{d}} \Phi\left(|\xi|^{2}\right)(\mathcal{F} f)^{2}(\xi) \mathrm{d} \xi, \quad f \in \mathcal{D}(\mathcal{E}),
$$

where $f \in \mathcal{D}(\mathcal{E})$ if and only both of $f(\xi)$ and $\sqrt{\Phi\left(|\xi|^{2}\right)} \mathcal{F} f(\xi)$ are in $L^{2}\left(\mathbb{R}^{d}\right)$. Note that the choice $\Phi(\lambda)=b \lambda, b>0$, leads to the only pure local operator in the class we consider, i.e. the operator $-b \Delta$. Whenever the Lévy measure $\rho$ in (2.1) is non-zero, the resulting operator $\Phi(-\Delta)$ is a non-local integral operator (for $b=0$ ) or an integro-differential operator (for $b>0$ ).

The semigroup $\left\{P_{t}: t \geqslant 0\right\}$ is the transition semigroup (and $-\Phi(-\Delta)$ is the generator) of a Markov process $X=\left(X_{t}\right)_{t \geqslant 0}$ which is determined by

$$
X_{t}=Z_{S_{t}}, \quad t \geqslant 0 .
$$

Such a process is obtained by a random time change of the Brownian motion $Z$ - this procedure is called the subordination. A new, random, clock of the process is given by the subordinator $S$ (we always assume that $Z$ and $S$ are independent). The process $X$ is referred to as the subordinate Brownian motion in $\mathbb{R}^{d}$. It is an isotropic Lévy process [36] with càdlàg paths whose Lévy-Khintchine exponent is equal to $\Phi\left(|\xi|^{2}\right)$. More precisely, we have

$$
\mathbf{E}_{0} \mathrm{e}^{i \xi \cdot X_{t}}=\mathrm{e}^{-t \Phi\left(|\xi|^{2}\right)}, \quad \xi \in \mathbb{R}^{d}, \quad t>0 .
$$

By $\mathbf{P}_{x}$ and $\mathbf{E}_{x}$ we denote the probability measure and the corresponding expected value for the process $X$ starting from $x \in \mathbb{R}^{d}$. We have $\mathbf{P}^{x}\left(X_{t} \in A\right)=\int_{A} p_{t}(x, y) \mathrm{d} y, A \in \mathcal{B}\left(\mathbb{R}^{d}\right)$, $x \in \mathbb{R}^{d}, t>0$, i.e. the kernels $p_{t}(x, y)$ are transition probability densities of the process $X$. It is important that under (1.6) we also have $\lim _{|\xi| \rightarrow \infty} \Phi\left(|\xi|^{2}\right) / \log |\xi|=\infty$, and it follows from [18, Lemma 2.1] that $(t, x) \mapsto p_{t}(x)$ is a continuous function on $(0, \infty) \times \mathbb{R}^{d}$.

Our Assumption (B) is satisfied by a wide class of complete Bernstein functions (and corresponding subordinators). Below we discuss only several, the most popular examples. For further examples we refer the reader e.g. to the monograph [35]. 


\section{Example 2.2.}

(1) Pure drift. Let $\Phi(\lambda)=b \lambda, b>0$. As mentioned above, this leads to the only subordinate Brownian motion with continuous paths - the Brownian motion with speed $b$.

(2) $\alpha / 2$-stable subordinators. Let $\Phi(\lambda)=\lambda^{\alpha / 2}, \alpha \in(0,2)$. The subordination via this subordinator leads to the pure jump isotropic $\alpha$-stable process.

(3) Mixture of several purely jump stable subordinators. In this case, $\Phi(\lambda)=\sum_{i=1}^{n} \lambda^{\alpha_{i} / 2}$, $\alpha_{i} \in(0,2), n \in \mathbb{Z}_{+}$.

(4) $\alpha / 2$-stable subordinator with drift. Let $\Phi(\lambda)=b \lambda+\lambda^{\alpha / 2}, \alpha \in(0,2), b>0$. Clearly, in this case, $\Phi(\lambda) \approx \lambda$ for $\lambda \rightarrow 0^{+}$, and $\Phi(\lambda) \approx \lambda^{\alpha / 2}$ for $\lambda \rightarrow \infty$.

(5) Relativistic $\vartheta / 2$-stable subordinator. Let $\Phi(\lambda)=\left(\lambda+m^{2 / \vartheta}\right)^{\vartheta / 2}-m, \vartheta \in(0,2), m>0$. The subordination via such a subordinator leads to the so-called relativistic $\vartheta$-stable process. Similarly as above, we have $\Phi(\lambda) \approx \lambda$ for $\lambda \rightarrow 0^{+}$, and $\Phi(\lambda) \approx \lambda^{\vartheta / 2}$ for $\lambda \rightarrow \infty$.

(6) If $S$ is a subordinator with Laplace exponent $\Phi(\lambda)=\lambda^{\alpha / 2}[\log (1+\lambda)]^{\beta / 2}, \alpha \in(0,2)$, $\beta \in(-\alpha, 0)$ or $\beta \in(0,2-\alpha)$, then we see that both the conditions (1.6) and (1.7) hold as well.

Next, we introduce the bridge measures of the subordinate process that will be needed in our argument. For fixed $t>0$ and $x, y \in \mathbb{R}^{d}$, the bridge measure $\mathbf{P}_{x, y}^{t}$ is defined by the following property: for any $0<s<t$ and $A \in \sigma\left(X_{u}: u \leqslant s\right)$,

$$
\mathbf{P}_{x, y}^{t}[A]=\frac{1}{p_{t}(x, y)} \mathbf{E}_{x}\left[\mathbf{1}_{A} p_{t-s}\left(X_{s}, y\right)\right]
$$

which is then extended to $s=t$ by weak continuity. The bridge measures can be understood as the laws of the process that starts from $x$ and is conditioned to have $X_{t}=y, \mathbf{P}_{x}$-almost surely. For more detailed information on Markovian bridges we refer to [6].

2.3. The operators and the corresponding subordinate processes on tori. Our argument in the present paper mostly uses subordinate semigroups and the related processes on the torus $\mathcal{T}_{M}$, for any $M \in \mathbb{Z}_{+}$. The torus $\mathcal{T}_{M}=\mathbb{R}^{d} /\left(M \mathbb{Z}_{+}\right)$is understood as the box $[0, M)^{d}$ with reciprocal sides identified. By $\pi_{M}$ we denote the canonical projection of $\mathbb{R}^{d}$ onto $\mathcal{T}_{M}$.

Let $\left\{G_{t}^{M}: t \geqslant 0\right\}$ be the heat semigroup acting on $L^{2}\left(\mathcal{T}_{M}\right)$, i.e.

$$
G_{t}^{M} f(x)=\int_{\mathcal{T}_{M}} g_{t}^{M}(x, y) f(y) \mathrm{d} y, \quad f \in L^{2}\left(\mathcal{T}_{M}\right), \quad t>0,
$$

where

$$
g_{t}^{M}(x, y):=\sum_{y^{\prime} \in \pi_{M}^{-1}(y)} g_{t}\left(x, y^{\prime}\right)=\sum_{\mathbf{i} \in M \mathbb{Z}^{d}} g_{t}(x, y+\mathbf{i}), t>0
$$

is the transition density of the Brownian motion on the torus $\mathcal{T}_{M}$ (in this formula, $g_{t}(x, y):=$ $g_{t}(y-x)$ denotes the classical Gauss-Weierstrass kernel). One can check that $G_{t}^{M}$ form a strongly continuous semigroup of bounded operators in $L^{2}\left(\mathcal{T}_{M}\right)$ (the latter fact is an easy consequence of the symmetry $g_{t}^{M}(x, y)=g_{t}^{M}(y, x)$ ). The infinitesimal generator of this semigroup (denoted by $\Delta^{M}$ ) is an unbounded, self-adjoint operator on $L^{2}\left(\mathcal{T}_{M}\right)$. It is important for our applications that the operators $G_{t}^{M}$ have certain scaling property: since $g_{a^{2} t}(a x)=g_{t}(x), a>0$, we also have

$$
g_{k^{2} t}^{k M}(k x, k y)=g_{t}^{M}(x, y), \quad x, y \in \mathcal{T}_{M}, t>0, k \in \mathbb{Z}_{+} .
$$

The subordinate heat semigroup on the torus is defined in the same way as its free counterpart in $\mathbb{R}^{d}$. For a Bernstein function $\Phi$ such that $\lim _{\lambda \searrow 0} \Phi(\lambda)=0$ and the convolution semigroup of measures $\left\{\eta_{t}: t \geqslant 0\right\}$ determined by (2.2), we let

$$
P_{t}^{M} f(x):=\int_{[0, \infty)} G_{u}^{M} f(x) \eta_{t}(\mathrm{~d} u), \quad f \in L^{2}\left(\mathcal{T}_{M}\right), t \geqslant 0
$$


$P_{t}^{M}$ form a strongly continuous semigroup of bounded self-adjoint operators in $L^{2}\left(\mathcal{T}_{M}\right)$. Under the assumption (1.6) all the $P_{t}$ 's, $t>0$, are integral operators with kernels given by

$$
p_{t}^{M}(x, y)=\int_{0}^{\infty} g_{u}^{M}(x, y) \eta_{t}(\mathrm{~d} u), \quad t>0 .
$$

Due to Fubini-Tonelli we have

$$
p_{t}^{M}(x, y)=\sum_{y^{\prime} \in \pi_{M}^{-1}(y)} \int_{0}^{\infty} g_{u}\left(x, y^{\prime}\right) \eta_{t}(\mathrm{~d} u)=\sum_{y^{\prime} \in \pi_{M}^{-1}(y)} p_{t}\left(x, y^{\prime}\right)=\sum_{\mathbf{i} \in M \mathbb{Z}^{d}} p_{t}(x, y+\mathbf{i}) .
$$

We have $P_{t}^{M}=\mathrm{e}^{-t \Phi\left(-\Delta^{M}\right)}$, where both the operators $\Phi\left(-\Delta^{M}\right)$ and $\mathrm{e}^{-t \Phi\left(-\Delta^{M}\right)}$ are understood through the spectral representation of unbounded self-adjoint operators.

As shown in Lemma 2.3 below, for every fixed $t>0$ both the kernels $g_{t}^{M}(x, y)$ and $p_{t}^{M}(x, y)$ are bounded functions on $\mathcal{T}_{M} \times \mathcal{T}_{M}$. Together with the fact that $\left|\mathcal{T}_{M}\right|=M^{d}<\infty$, this gives that the operators $G_{t}^{M}$ and $P_{t}^{M}$ are Hilbert-Schmidt on $L^{2}\left(\mathcal{T}_{M}\right)$. In consequence, all the operators considered in this section have purely discrete spectral decompositions. Indeed, for $M=1,2, \ldots$, the spectrum of the operator $-\Delta^{M}$ consists of a sequence of eigenvalues

$$
0 \leqslant \mu_{1}^{M}<\mu_{2}^{M} \leqslant \mu_{3}^{M} \leqslant \ldots \rightarrow \infty,
$$

each of finite multiplicity, and the corresponding eigenfunctions $\left\{\psi_{k}^{M}\right\}_{k=1}^{\infty}$ form a complete orthonormal system in $L^{2}\left(\mathcal{T}_{M}\right)$. We have

$$
-\Delta^{M} \psi_{k}^{M}=\mu_{k}^{M} \psi_{k}^{M} \quad \text { and } \quad G_{t}^{M} \psi_{k}^{M}=\mathrm{e}^{-t \mu_{k}^{M}} \psi_{k}^{M}, \quad t>0, \quad k=1,2, \ldots,
$$

and due to the conservativeness of the semigroup $\left\{G_{t}^{M}: t \geqslant 0\right\}$,

$$
\mu_{1}^{M}=0 \quad \text { and } \quad \psi_{1}^{M} \equiv \frac{1}{\sqrt{\left|\mathcal{T}_{M}\right|}}=\frac{1}{M^{d / 2}} .
$$

One can directly check that the eigenvalues of the operators $-\Delta^{M}$ inherit from (2.5) the following scaling property:

$$
\mu_{k}^{M}=M^{-2} \mu_{k}^{1}, \quad k=1,2,3, \ldots
$$

Due to the spectral theorem, the spectrum of the operator $\Phi\left(-\Delta^{M}\right)$ consists of eigenvalues $\lambda_{1}^{M}<\lambda_{2}^{M} \leqslant \lambda_{3}^{M} \leqslant \ldots \rightarrow \infty$ satisfying

$$
\lambda_{1}^{M}=0 \quad \text { and } \quad \lambda_{k}^{M}=\Phi\left(\mu_{k}^{M}\right), \quad k=2,3, \ldots,
$$

and the corresponding eigenfunctions are exactly the same as above. More precisely, we have

$$
\Phi\left(-\Delta^{M}\right) \psi_{k}^{M}=\lambda_{k}^{M} \psi_{k}^{M} \quad \text { and } \quad P_{t}^{M} \psi_{k}^{M}=\mathrm{e}^{-t \lambda_{k}^{M}} \psi_{k}^{M}, \quad t>0, \quad k=1,2, \ldots
$$

Our present work requires additional regularity properties of the kernels $p_{t}^{M}(x, y)$ such as continuity, boundedness, and on-diagonal estimates, gathered in the following Lemma.

Lemma 2.3. The following hold.

(1) There exists a constant $C>0$ such that for every $M, n \in \mathbb{Z}_{+}, x, y \in[0, M)^{d}$ and $t>0$ we have

$$
\sum_{\substack{\mathbf{i} \in M \mathbb{Z}^{d} \\ \mathbf{i} \notin[-n M, n M]^{d}}} g_{t}(x, y+\mathbf{i}) \leqslant \frac{C}{M^{d}}\left(\frac{M n}{\sqrt{t}} \vee 1\right)^{d-2} \mathrm{e}^{-\frac{1}{16}\left(\frac{M n}{\sqrt{t}} \vee 1\right)^{2}} .
$$

In particular, the series defining the kernel $g_{t}^{M}(x, y)$ is uniformly convergent in $(t, x, y)$ on every cuboid $[u, v] \times[0, M)^{d} \times[0, M)^{d}, 0<u<v<\infty$, and there exists a universal constant $\widetilde{C}>0$ such that for every $M \in \mathbb{Z}_{+}$we have

$$
g_{t}^{M}(x, y) \leqslant \widetilde{C}\left(t^{-d / 2} \vee M^{-d}\right), \quad x, y \in \mathcal{T}_{M}, t>0 .
$$


(2) Under the assumption (1.6) the function $(t, x, y) \mapsto p_{t}^{M}(x, y)$ is continuous on $(0, \infty) \times \mathcal{T}_{M} \times \mathcal{T}_{M}$. Moreover, there exists a universal constant $C>0$ such that for every $M \in Z_{+}$we have

$$
p_{t}^{M}(x, y) \leqslant C\left(\int_{0}^{\infty} \mathrm{e}^{-t \Phi\left(\lambda^{2 / d}\right)} \mathrm{d} \lambda \vee M^{-d}\right), \quad x, y \in \mathcal{T}_{M}, t>0 .
$$

In particular, $p_{t}^{M}(x, y)$ is bounded on every cuboid $\left[t_{0}, \infty\right) \times \mathcal{T}_{M} \times \mathcal{T}_{M}, t_{0}>0$.

Proof. (1) For every $x, y \in[0, M)^{d}, t>0$ and $M, n \in \mathbb{Z}_{+}$we have

$$
\begin{aligned}
\sum_{\substack{\mathbf{i} \in M Z^{d} \\
\mathbf{i} \notin[-n M, n M]^{d}}} g_{t}(x, y+\mathbf{i}) & \leqslant \sum_{k \geqslant n} \frac{(2(k+1))^{d}-(2 k)^{d}}{(4 \pi t)^{d / 2}} \mathrm{e}^{-\frac{(k M)^{2}}{4 t}} \\
& \leqslant \frac{c}{\sqrt{t} M^{d-1}} \sum_{k \geqslant n}\left(\frac{k M}{\sqrt{t}}\right)^{d-1} \mathrm{e}^{-\left(\frac{(k+1) M}{4 \sqrt{t}}\right)^{2}} \\
& \leqslant \frac{c}{\sqrt{t} M^{d-1}} \int_{n}^{\infty}\left(\frac{M x}{\sqrt{t}}\right)^{d-1} \mathrm{e}^{-\frac{1}{16}\left(\frac{M x}{\sqrt{t}}\right)^{2}} \mathrm{~d} x,
\end{aligned}
$$

with an absolute constant $c>0$. By substitution, the latter expression is equal to

$$
\frac{c}{M^{d}} \int_{\frac{M n}{\sqrt{t}}}^{\infty} y^{d-1} \mathrm{e}^{-\frac{y^{2}}{16}} \mathrm{~d} y
$$

Using the elementary estimate

$$
\int_{a}^{\infty} y^{d-1} \mathrm{e}^{-\frac{y^{2}}{16}} \mathrm{~d} y \leqslant c_{1}(a \vee 1)^{d-2} \mathrm{e}^{-\frac{1}{16}(a \vee 1)^{2}}, \quad a>0,
$$

where $c_{1}>0$ is a uniform constant, we finally get

$$
\sum_{\substack{\mathbf{i} \in M \mathbb{Z}^{d} \\ \mathbf{i} \notin[-n M, n M]^{d}}} g_{t}(x, y+\mathbf{i}) \leqslant \frac{c_{2}}{M^{d}}\left(\frac{M n}{\sqrt{t}} \vee 1\right)^{d-2} \mathrm{e}^{-\frac{1}{16}\left(\frac{M n}{\sqrt{t}} \vee 1\right)^{2}},
$$

which is exactly the first assertion of part (1). The uniform convergence follows directly from this uniform bound for the tail of the series. To prove the other assertion of (1), we write

$$
g_{t}^{M}(x, y) \leqslant c_{3} t^{-d / 2}+\sum_{\substack{\mathbf{i} \in M \mathbb{Z}^{d} \\ \mathbf{i} \notin[-M, M]^{d}}} g_{t}(x, y+\mathbf{i}) .
$$

The second term can be easily estimated by using the bound proven above with $n=1$. We have two cases. If $\sqrt{t} \geqslant M$, then $g_{t}^{M}(x, y) \leqslant c_{3} t^{-d / 2}+c_{2} M^{-d} \leqslant\left(c_{2}+c_{3}\right) M^{-d}$. If $\sqrt{t}<M$, then

$$
\left(\frac{M}{\sqrt{t}} \vee 1\right)^{d-2} \mathrm{e}^{-\frac{1}{16}\left(\frac{M}{\sqrt{t}} \vee 1\right)^{2}}=\left(\frac{M}{\sqrt{t}}\right)^{d-2} \mathrm{e}^{-\frac{1}{16}\left(\frac{M}{\sqrt{t}}\right)^{2}} \leqslant c_{4},
$$

and, similarly as above, $g_{t}^{M}(x, y) \leqslant c_{3} t^{-d / 2}+c_{2} c_{4} M^{-d} \leqslant\left(c_{2} c_{4}+c_{3}\right) t^{-d / 2}$. This implies the second estimate in (1).

(2) We first show the estimate and the boundedness. By the upper estimate for the kernel $g_{t}^{M}(x, y)$ proven above, for $M \in \mathbb{Z}_{+}, x, y \in \mathcal{T}_{M}$, and $t>0$, we have

$$
p_{t}^{M}(x, y) \leqslant c_{4}\left(\int_{0}^{M^{2}} u^{-d / 2} \eta_{t}(\mathrm{~d} u)+M^{-d} \eta_{t}\left[M^{2}, \infty\right)\right) \leqslant c_{4}\left(\int_{0}^{\infty} u^{-d / 2} \eta_{t}(\mathrm{~d} u)+M^{-d}\right) .
$$

We then derive from Lemma 2.1 that

$$
p_{t}^{M}(x, y) \leqslant c_{5}\left(\int_{0}^{\infty} \mathrm{e}^{-t \Phi\left(\lambda^{2 / d}\right)} \mathrm{d} \lambda+M^{-d}\right)
$$


and, for every $t_{0}>0$,

$$
\sup _{(t, x, y) \in\left[t_{0}, \infty\right) \times \mathcal{T}_{M} \times \mathcal{T}_{M}} p_{t}^{M}(x, y)<\infty .
$$

We now prove the continuity. Since the function $(t, x, y) \mapsto p_{t}(x, y)$ is continuous on $(0, \infty) \times \mathbb{R}^{d} \times \mathbb{R}^{d}$, it is enough to justify that the series

$$
\sum_{\mathbf{i} \in M \mathbb{Z}^{d}} p_{t}(x, y+\mathbf{i})
$$

is uniformly convergent on every cuboid $\left[t_{0}, t_{1}\right] \times[0, M)^{d} \times[0, M)^{d}, 0<t_{0}<t_{1}<\infty$. We only need to prove that the tail

$$
\sum_{\substack{\mathbf{i} \in M \mathbb{Z}^{d} \\ \mathbf{i} \notin[-n M, n M]^{d}}} p_{t}(x, y+\mathbf{i})
$$

goes to zero as $n \rightarrow \infty$, uniformly in $(t, x, y) \in\left[t_{0}, t_{1}\right] \times[0, M)^{d} \times[0, M)^{d}$. Using the tail estimate from part (1), Fubini-Tonelli an the fact that $c_{6}:=\sup _{r \geqslant 1} r^{d-2} \mathrm{e}^{-r^{2} / 16}<\infty$, we get

$$
\begin{gathered}
\sum_{\substack{\mathbf{i} \in M \mathbb{Z}^{d} \\
\mathbf{i} \notin[-n M, n M]^{d}}} p_{t}(x, y+\mathbf{i}) \leqslant \frac{c_{7}}{M^{d}} \int_{0}^{\infty}\left(\frac{M n}{\sqrt{u}} \vee 1\right)^{d-2} \mathrm{e}^{-\frac{1}{16}\left(\frac{M n}{\sqrt{u}} \vee 1\right)^{2}} \eta_{t}(\mathrm{~d} u) \\
\leqslant \frac{c_{7}}{M^{d}}\left(\int_{(0, n]}\left(\frac{M n}{\sqrt{u}}\right)^{d-2} \mathrm{e}^{-\frac{1}{16}\left(\frac{M n}{\sqrt{u}}\right)^{2}} \eta_{t}(\mathrm{~d} u)+c_{6} \eta_{t}(n, \infty)\right) \\
\leqslant \frac{c_{7}}{M^{2}} n^{d-1} \mathrm{e}^{-\frac{n}{16}} \int_{(0, n]} u^{-d / 2} \eta_{t}(\mathrm{~d} u)+c_{6} c_{7} \eta_{t}(n, \infty) .
\end{gathered}
$$

Now, by Lemma 2.1, the integral $\int_{0}^{\infty} u^{-d / 2} \eta_{t}(\mathrm{~d} u)$ is uniformly bounded for $t \geqslant t_{0}$. Moreover, by following the argument in [15, Lemma 2.2], we obtain

$$
\int_{0}^{n} \mathrm{e}^{-\lambda u} \eta_{t}(u, \infty) \mathrm{d} u \leqslant \frac{t \Phi(\lambda)}{\lambda}, \quad \lambda, t>0
$$

which yields

$$
\eta_{t}(n, \infty)\left(1-\mathrm{e}^{-\lambda n}\right) \leqslant t \Phi(\lambda), \quad \lambda, t>0 .
$$

By taking $\lambda=1 / n$, we get $\eta_{t}(n, \infty) \leqslant\left(1-\mathrm{e}^{-1}\right)^{-1} t \Phi(1 / n) \leqslant\left(1-\mathrm{e}^{-1}\right)^{-1} t_{1} \Phi(1 / n)$, whenever $t \leqslant t_{1}$. This implies the claimed uniform convergence, completing the proof of the lemma.

The semigroup $\left\{P_{t}^{M}: t \geqslant 0\right\}$ determines a conservative Markov process $\left(X_{t}^{M}\right)_{t \geqslant 0}$ on the torus $\mathcal{T}_{M}$. If we denote by $\mathbf{P}_{x}^{M}$ the measure concentrated on trajectories that start from $x \in \mathcal{T}_{M}$, then

$$
\mathbf{P}_{x}^{M}\left(X_{t}^{M} \in A\right)=P_{t}^{M} \mathbb{1}_{A}(x)=\int_{A} p_{t}^{M}(x, y) \mathrm{d} y, \quad A \in \mathcal{B}\left(\mathcal{T}_{M}\right), x \in \mathcal{T}_{M}, t>0 .
$$

It is a symmetric Feller process with continuous and bounded transition probability densities $p_{t}^{M}(x, y)$. Due to (2.6) this process can be identified pathwise as

$$
X_{t}^{M}=\pi_{M}\left(X_{t}\right), \quad t>0,
$$

where $\left(X_{t}\right)_{t \geqslant 0}$ is the subordinate Brownian motion in $\mathbb{R}^{d}$, introduced in the previous section. Throughout the paper we call this process the subordinate Brownian motion on the torus $\mathcal{T}_{M}$.

For given $t \geqslant 0$ and $x, y \in \mathcal{T}_{M}$, the bridge measure of the process $X^{M}$, conditioned to have $X_{t}^{M}=y, \mathbf{P}_{x}^{M}$-almost surely, is defined by a relation similar to (2.4). These measures are denoted by $\mathbf{P}_{x, y}^{M, t}$.

The bridge measures for the process in $\mathbb{R}^{d}$ and on the torus $\mathcal{T}_{M}$ are related through the following identity. 
Lemma 2.4. For every $t>0, x, y \in \mathbb{R}^{d}, M=1,2, \ldots$ and any set $A \in \mathcal{B}\left(D\left([0, t], \mathcal{T}_{M}\right)\right.$ we have

$$
p_{t}^{M}\left(\pi_{M}(x), \pi_{M}(y)\right) \mathbf{P}_{\pi_{M}(x), \pi_{M}(y)}^{M, t}[A]=\sum_{y^{\prime} \in \pi_{M}^{-1}\left(\pi_{M}(y)\right)} p_{t}\left(x, y^{\prime}\right) \mathbf{P}_{x, y^{\prime}}^{t}\left[\pi_{M}^{-1}(A)\right]
$$

$\left(D\left([0, t], \mathcal{T}_{M}\right)\right)$ is the Skorohod space).

This statement is readily seen for cylindrical sets and then extended to the desired range of $A$ 's by the Monotone Class Theorem. Its fractal counterpart was discussed in [15, Lemma 2.6].

2.4. Random Anderson (alloy-type) potentials. Our approach in the present paper allows us to study the alloy-type random fields

$$
V^{\omega}(x)=\sum_{\mathbf{i} \in \mathbb{Z}^{d}} q_{\mathbf{i}}(\omega) W(x-\mathbf{i}), \quad x \in \mathbb{R}^{d},
$$

with possibly singular single-site potentials $W$ of bounded support which are in Kato classes corresponding to the operators considered. The main part of our argument is based on an application of certain periodization of such potentials: for given $M \geqslant 1$ we define

$$
\begin{aligned}
V_{M}^{\omega}(x) & :=\sum_{\mathbf{i} \in[0, M)^{d}}\left(q_{\mathbf{i}}(\omega) \sum_{\mathbf{i}^{\prime} \in \pi_{M}^{-1}(\mathbf{i})} W\left(x-\mathbf{i}^{\prime}\right)\right) \\
& =\sum_{\mathbf{i} \in \mathbb{Z}^{d}} q_{\pi_{M}(\mathbf{i})} W(x-\mathbf{i}), \quad x \in \mathbb{R}^{d} .
\end{aligned}
$$

This means that we first periodize the lattice random variables $\left\{q_{\mathbf{i}}\right\}_{\mathbf{i} \in \mathbb{Z}^{d}}$ with respect to $\pi_{M}$, and then, based on that, we construct a new random potential which is also periodic in the usual sense: $V_{M}^{\omega}(x+\mathbf{i})=V_{M}^{\omega}(x), \mathbf{i} \in M \mathbb{Z}^{d}$. We call it the Sznitman-type periodization of $V^{\omega}$. For simplicity, we will use the same letter for the restriction of this potential to $\mathcal{T}_{M}$.

Recall that the Kato class $\mathcal{K}$ associated with the operator $\Phi(-\Delta)$ consists of those Borel functions $f: \mathbb{R}^{d} \rightarrow \mathbb{R}$ for which

$$
\lim _{t \searrow 0} \sup _{x \in \mathbb{R}^{d}} \int_{0}^{t} P_{s}|f|(x) \mathrm{d} s=0 .
$$

Similarly, a Borel function $f: \mathcal{T}_{M} \rightarrow \mathbb{R}$ belongs to the Kato class $\mathcal{K}^{M}$ associated with $\Phi\left(-\Delta^{M}\right)$ if

$$
\lim _{t \searrow 0} \sup _{x \in \mathcal{T}_{M}} \int_{0}^{t} P_{s}^{M}|f|(x) \mathrm{d} s=0 .
$$

Moreover, we say that a Borel function $f$ belongs to the local Kato class $\mathcal{K}_{\text {loc }}$ if its restriction to an arbitrary bounded Borel subset of $\mathbb{R}^{d}$ is in $\mathcal{K}$. Note that the torus $\mathcal{T}_{M}$ is a compact space and so the local Kato class for $\mathcal{T}_{M}$ would agree with $\mathcal{K}^{M}$. Therefore there is no need to define it separately. One can check that $L^{\infty}\left(\mathbb{R}^{d}\right) \subset \mathcal{K}$ and $L_{\text {loc }}^{\infty}\left(\mathbb{R}^{d}\right) \subset \mathcal{K}_{\text {loc }}$. Moreover, $\mathcal{K}_{\text {loc }} \subset L_{\text {loc }}^{1}\left(\mathbb{R}^{d}\right)$ and $\mathcal{K}^{M} \subset L^{1}\left(\mathcal{T}_{M}\right)=L_{\text {loc }}^{1}\left(\mathcal{T}_{M}\right)$.

We now show that the alloy-type random potentials $V^{\omega}$ and $V_{M}^{\omega}$ inherit the Kato-regularity from their profiles $W$.

Proposition 2.5. Let $W \in \mathcal{K}, W \geqslant 0$, be of bounded support and let the assumption (Q) hold. Then, for every $M \in \mathbb{Z}_{+}$and $\omega \in \Omega$, we have

(1) $V^{\omega} \in \mathcal{K}_{\text {loc }}$,

(2) $V_{M}^{\omega} \in \mathcal{K}_{\text {loc }}$

(3) $V_{M}^{\omega} \in \mathcal{K}^{M}$. 
Proof. Fix $\omega \in \Omega$ and suppose $\operatorname{supp} W \subset\left[-M_{0}, M_{0}\right]^{d}$, for some $M_{0} \in \mathbb{Z}_{+}$.

(1) Denote $V^{n, \omega}=\mathbb{1}_{[-n, n]^{d}} V^{\omega}, n \in \mathbb{Z}_{+}$. Observe that

$$
V^{n, \omega}(x) \leqslant \sum_{\mathbf{i} \in \mathbb{Z}^{d} \cap\left[-M_{0}-n, M_{0}+n\right]^{d}} q_{\mathbf{i}}(\omega) W(x-\mathbf{i}), \quad x \in \mathbb{R}^{d} .
$$

We have

$$
\begin{aligned}
\int_{0}^{t} P_{s} V^{n, \omega}(x) \mathrm{d} s & \leqslant \sum_{\mathbf{i} \in \mathbb{Z}^{d} \cap\left[-M_{0}-n, M_{0}+n\right]^{d}} q_{\mathbf{i}}(\omega) \int_{0}^{t} P_{s} W(x-\mathbf{i}) \mathrm{d} s \\
& \leqslant \sup _{y \in \mathbb{R}^{d}} \int_{0}^{t} P_{s} W(y)\left(\sum_{\mathbf{i} \in \mathbb{Z}^{d} \cap\left[-M_{0}-n, M_{0}+n\right]^{d}} q_{\mathbf{i}}(\omega)\right) \mathrm{d} s .
\end{aligned}
$$

The sum on the right hand side has finitely many terms and $W \in \mathcal{K}$. Therefore by taking the supremum over $x \in \mathbb{R}^{d}$ on the left hand side and then letting $t \searrow 0$, we get that

$$
\sup _{x \in \mathbb{R}^{d}} \int_{0}^{t} P_{s} V^{n, \omega}(x) \rightarrow 0,
$$

for arbitrary $n \in \mathbb{Z}_{+}$. Hence $V^{\omega} \in \mathcal{K}_{\text {loc }}$.

(2) The proof is a minor modification of that of (1) as we only need to replace $q_{\mathbf{i}}(\omega)$ with $q_{\pi_{M}(\mathbf{i})}(\omega)$ in the sum defining the potential.

(3) Fix $M \in \mathbb{Z}_{+}$. By the definition of the operators $P_{t}^{M}$ and the potential $V_{M}^{\omega}$, for every $x \in \mathcal{T}_{M}$ and $s>0$ we have

$$
\begin{aligned}
P_{s}^{M} V_{M}^{\omega}(x) & =\int_{\mathcal{T}_{M}} p_{s}^{M}(x, y) V_{M}^{\omega}(y) \mathrm{d} y \\
& =\int_{[0, M)^{d}}\left(\sum_{\mathbf{i} \in M \mathbb{Z}^{d}} p_{t}(x, y+\mathbf{i})\right) V_{M}^{\omega}(y) \mathrm{d} y \\
& =\int_{[0, M)^{d}}\left(\sum_{\substack{\mathbf{i} \in M \mathbb{Z}^{d} \\
\mathbf{i} \in[-M, M]^{d}}} p_{t}(x, y+\mathbf{i})+\sum_{\substack{\mathbf{i} \in M \mathbb{Z}^{d} \\
\mathbf{i} \notin[-M, M]^{d}}} p_{t}(x, y+\mathbf{i})\right) V_{M}^{\omega}(y) \mathrm{d} y \\
& =P_{t}\left(\mathbb{1}_{[-M, M]^{d}} V_{M}^{\omega}\right)(x)+\int_{[0, M)^{d}}\left(\sum_{\substack{\mathbf{i} \in M \mathbb{Z}^{d} \\
\mathbf{i} \notin[-M, M]^{d}}} p_{t}(x, y+\mathbf{i})\right) V_{M}^{\omega}(y) \mathrm{d} y .
\end{aligned}
$$

By Fubini-Tonelli and the tail estimate in Lemma 2.3 (1)

$$
\sum_{\substack{\mathbf{i} \in M \mathbb{Z}^{d} \\ \mathbf{i} \notin[-M, M]^{d}}} p_{t}(x, y+\mathbf{i})=\int_{0}^{\infty}\left(\sum_{\substack{\mathbf{i} \in M \mathbb{Z}^{d} \\ \mathbf{i} \notin[-M, M]^{d}}} g_{t}(x, y+\mathbf{i})\right) \eta_{t}(\mathrm{~d} u) \leqslant c M^{-d},
$$

which gives

$$
P_{s}^{M} V_{M}^{\omega}(x) \leqslant P_{t}\left(\mathbb{1}_{[-M, M]^{d}} V_{M}^{\omega}\right)(x)+c M^{-d} \int_{[0, M)^{d}} V_{M}^{\omega}(y) \mathrm{d} y .
$$

By part (2) we have $V_{M}^{\omega} \in \mathcal{K}_{\text {loc }}$. In particular, $V_{M}^{\omega} \in L_{\text {loc }}^{1}\left(\mathbb{R}^{d}\right)$. Hence

$$
\sup _{x} \int_{0}^{t} P_{s}^{M} V_{M}^{\omega}(x) \mathrm{d} s \leqslant \sup _{x} \int_{0}^{t} P_{t}\left(\mathbb{1}_{[-M, M]^{d}} V_{M}^{\omega}\right)(x) \mathrm{d} s+c t M^{-d} \int_{[0, M)^{d}} V_{M}^{\omega}(y) \mathrm{d} y \longrightarrow 0,
$$

as $t \searrow 0$. This means that $V_{M}^{\omega} \in \mathcal{K}^{M}$. 
As mentioned in the introduction, every bounded function with compact support is automatically in the Kato class $\mathcal{K}$. We now provide examples of singular functions from $\mathcal{K}$.

Example 2.6. Let $\Phi(\lambda)=\lambda^{\alpha / 2}, \alpha \in(0,2]$ (i.e. we either consider the Laplace operator $-\Delta$ or the fractional Laplace operators $\left.(-\Delta)^{\alpha / 2}, \alpha \in(0,2)\right)$. For simplicity, assume additionally that $\alpha<d \in \mathbb{Z}_{+}$. It is known (see e.g. [2]) that in this case

$$
f \in \mathcal{K} \quad \Longleftrightarrow \quad \lim _{r \searrow 0} \sup _{x \in \mathbb{R}^{d}} \int_{|x-y|<r} \frac{f(y)}{|x-y|^{d-\alpha}} \mathrm{d} y=0 .
$$

The same is true for $\Phi(\lambda)=\left(\lambda+m^{2 / \alpha}\right)^{\alpha / 2}-m, \alpha \in(0,2), m>0$, i.e. for the quasi-relativistic operators. If we now take $W(y):=\mathbb{1}_{B(0,1)}(y)|y|^{-\beta}, \beta>0$, then we see that $W \in \mathcal{K}$ if and only if $\beta<\alpha$. In view of the assumption (W) it is also instructive to verify that $W \in L^{2}\left(\mathbb{R}^{d}\right)$ if and only if $\beta<d / 2$. In particular, $W \in \mathcal{K} \cap L^{2}\left(\mathbb{R}^{d}\right)$ if and only if $\beta<\alpha \wedge d / 2$.

This example indicates that the intersection $\mathcal{K} \cap L^{2}\left(\mathbb{R}^{d}\right)$ is typically a fairly non-trivial function space, but in general there are no inclusions between $\mathcal{K}$ and $L^{2}\left(\mathbb{R}^{d}\right)$.

2.5. Schrödinger operators and the Feynman-Kac formula. We now introduce the class of random Schrödinger operators based on $\Phi(-\Delta)$ and $-\Phi\left(-\Delta^{M}\right)$, and we discuss their spectral properties. Our standard reference here will be the monograph of Demuth and van Casteren [9] which is concerned with the spectral theory of self-adjoint Feller operators.

In the previous sections we have verified that the subordinate semigroups $\left\{P_{t}: t \geqslant 0\right\}$ and $\left\{P_{t}^{M}: t \geqslant 0\right\}$, determined by the kernels $p_{t}(x, y)$ and $p_{t}^{M}(x, y)$, respectively, satisfy the basic assumptions of spectral stochastic analysis (BASSA in short) and in consequence the operators $-\Phi(-\Delta)$ and $-\Phi\left(-\Delta^{M}\right)$ are (free) Feller generators [9, Assumptions A1-A4 and Definition 1.3 in Section 1.B].

Throughout this section we assume that $V^{\omega}$ and $V_{M}^{\omega}$ are random alloy-type potentials given by (2.11) and (2.12), constructed for a compactly supported and nonnegative single-site potential $W \in \mathcal{K}$ and lattice random variables $\left\{q_{\mathbf{i}}\right\}_{\mathbf{i} \in \mathbb{Z}^{d}}$ satisfying the assumption (Q). Thus, by Proposition 2.5, we have $V^{\omega} \in \mathcal{K}_{\text {loc }}$ and $V_{M}^{\omega} \in \mathcal{K}^{M}$, for every realization of lattice configuration. This allows us to define the random Schrödinger operators

$$
H^{\omega}=\Phi(-\Delta)+V^{\omega} \quad \text { and } \quad H_{M}^{\omega}=\Phi\left(-\Delta^{M}\right)+V_{M}^{\omega}
$$

as positive self-adjoint operators on $L^{2}\left(\mathbb{R}^{d}\right)$ and $L^{2}\left(\mathcal{T}_{M}\right)$, respectively [9, Theorem 2.5]. It is decisive for this work that the evolution semigroups of these operators can be represented probabilistically with respect to subordinate processes $\left(X_{t}\right)_{t \geqslant 0}$ and $\left(X_{t}^{M}\right)_{t \geqslant 0}$. More precisely, the following Feynman-Kac formulas hold:

$$
P_{t}^{V^{\omega}} f(x):=\mathrm{e}^{-t H^{\omega}} f(x)=\mathbf{E}_{x}\left[\mathrm{e}^{-\int_{0}^{t} V^{\omega}\left(X_{s}\right) \mathrm{d} s} f\left(X_{t}\right)\right], \quad f \in L^{2}\left(\mathbb{R}^{d}\right), \quad t>0,
$$

and

$$
P_{t}^{M, V_{M}^{\omega}} f(x):=\mathrm{e}^{-t H_{M}^{\omega}} f(x)=\mathbf{E}_{x}^{M}\left[\mathrm{e}^{-\int_{0}^{t} V_{M}^{\omega}\left(X_{s}^{M}\right) \mathrm{d} s} f\left(X_{t}^{M}\right)\right], \quad f \in L^{2}\left(\mathcal{T}_{M}\right), \quad t>0 .
$$

Both $P_{t}^{V^{\omega}}$ and $P_{t}^{M, V_{M}^{\omega}}, t>0$, are integral operators with bounded and symmetric kernels

$$
p_{t}^{V^{\omega}}(x, y)=p_{t}(x, y) \mathbf{E}_{x, y}^{t}\left[\mathrm{e}^{-\int_{0}^{t} V^{\omega}\left(X_{s}\right) \mathrm{d} s}\right]
$$

and

$$
p_{t}^{M, V^{\omega}}(x, y)=p_{t}^{M}(x, y) \mathbf{E}_{x, y}^{M, t}\left[\mathrm{e}^{-\int_{0}^{t} V_{M}^{\omega}\left(X_{s}^{M}\right) \mathrm{d} s}\right],
$$

where $\mathbf{E}_{x, y}^{t}$ and $\mathbf{E}_{x, y}^{M, t}$ are expected values with respect to bridge measures $\mathbf{P}_{x, y}^{t}$ and $\mathbf{P}_{x, y}^{M, t}$ introduced in previous sections. 
Observe that $p_{t}^{M, V^{\omega}}(x, y) \leqslant p_{t}^{M}(x, y)$ and recall that for every fixed $t>0$ the kernel $p_{t}^{M}(x, y)$ is a bounded function on $\mathcal{T}_{M} \times \mathcal{T}_{M}$. Since $\left|\mathcal{T}_{M}\right|=M^{d}<\infty$, this gives that the operators $P_{t}^{M, V_{M}^{\omega}}$, $t>0$, are Hilbert-Schmidt in $L^{2}\left(\mathcal{T}_{M}\right)$. This implies that the spectrum of the Schrödinger operator $H_{M}^{\omega}$ is discrete - it consists of a sequence of eigenvalues

$$
0 \leqslant \lambda_{1}^{M, V^{\omega}}<\lambda_{2}^{M, V^{\omega}} \leqslant \lambda_{3}^{M, V^{\omega}} \leqslant \ldots \rightarrow \infty
$$

with finite multiplicities, and the corresponding eigenfunctions $\left\{\psi_{k}^{M, V^{\omega}}\right\}_{k=1}^{\infty}$ form a complete orthonormal system in $L^{2}\left(\mathcal{T}_{M}\right)$.

2.6. Dirichlet Schrödinger operators and the integrated density of states. Denote by $H_{\Lambda}^{\omega}$ the operator $H^{\omega}$ constrained to a bounded, nonempty region $\Lambda \subset \mathbb{R}^{d}$ (we consider Dirichlet conditions on $\Lambda^{c}$ in the non-local case and on $\partial \Lambda$ in the local case) and let $\left\{\mathrm{e}^{-t H_{\Lambda}^{\omega}} ; t \geqslant 0\right\}$ be its evolution semigroup on $L^{2}(\Lambda)$. Then we have the following Feynman-Kac formula:

$$
\mathrm{e}^{-t H_{\Lambda}^{\omega}}=P_{t}^{V^{\omega}, \Lambda} f(x):=\mathbf{E}_{x}\left[\mathrm{e}^{-\int_{0}^{t} V^{\omega}\left(X_{s}\right) \mathrm{d} s} f\left(X_{t}\right) ; t<\tau_{\Lambda}\right], \quad f \in L^{2}(\Lambda, \mathrm{d} x), \quad t>0 .
$$

Here $\tau_{\Lambda}:=\inf \left\{t \geqslant 0: X_{t} \notin \Lambda\right\}$ denotes the first exit time of the process from the domain $\Lambda$. All the $P_{t}^{V^{\omega}, \Lambda}, t>0$, are integral operators with bounded and symmetric kernels

$$
p_{t}^{V^{\omega}, \Lambda}(x, y)=p_{t}(x, y) \mathbf{E}_{x, y}^{t}\left[\mathrm{e}^{-\int_{0}^{t} V^{\omega}\left(X_{s}\right) \mathrm{d} s} ; t<\tau_{\Lambda}\right] .
$$

Again, since $|\Lambda|<\infty$, the operators $P_{t}^{V^{\omega}, \Lambda}, t>0$, are Hilbert-Schmidt. In particular, there exists a complete orthonormal system, consisting of eigenfunctions of the operator $H_{\Lambda}^{\omega}$. The corresponding eigenvalues satisfy $0 \leqslant \lambda_{1}^{V^{\omega}}(\Lambda)<\lambda_{2}^{V^{\omega}}(\Lambda) \leqslant \lambda_{3}^{V^{\omega}}(\Lambda) \leqslant \ldots \rightarrow \infty$; each $\lambda_{k}^{V^{\omega}}(\Lambda)$ is of finite multiplicity and the ground state eigenvalue $\lambda_{1}^{V^{\omega}}(\Lambda)$ is simple.

We are now in a position to give the formal definition of the IDS. For a given bounded domain $\Lambda \subset \mathbb{R}^{d}$, let

$$
\ell_{\Lambda}^{\omega}(\cdot)=\frac{1}{|\Lambda|} \sum_{k=1}^{\infty} \delta_{\lambda_{k}^{V \omega}(\Lambda)}(\cdot)
$$

be the counting measure on the spectrum of $H_{\Lambda}^{\omega}$, normalized by the volume. Under the assumption (Q), the random alloy-type potential $V^{\omega}$ is stationary with respect to $\mathbb{Z}^{d}$. Therefore if we restrict our attention to sets $\Lambda$ composed of unit cubes with vertices in $\mathbb{Z}^{d}$, then it follows from the maximal ergodic theorem (see e.g. 4, Remark VI.1.2]) that the measures $\ell_{\Lambda}^{\omega}$ converge vaguely, as $\Lambda \nearrow \mathbb{R}^{d}$, to a nonrandom measure $\ell$, which is called the integrated density of states of $H^{\omega}$. The vague convergence of $\ell_{\Lambda}^{\omega}$ when $\Lambda \nearrow \mathbb{R}^{d}$ amounts to the convergence of their Laplace transforms

$$
\begin{aligned}
L_{\Lambda}^{\omega}(t) & =\frac{1}{|\Lambda|} \int_{[0, \infty)} \mathrm{e}^{-t \lambda} \ell_{\Lambda}^{\omega}(\mathrm{d} \lambda)=\frac{1}{|\Lambda|} \operatorname{Tr} P_{t}^{V^{\omega}, \Lambda}=\frac{1}{|\Lambda|} \int_{\Lambda} p_{t}^{V^{\omega}, \Lambda}(x, x) \mathrm{d} x \\
& =\frac{p_{t}(0,0)}{|\Lambda|} \int_{\Lambda} \mathbf{E}_{x, x}^{t}\left[\mathrm{e}^{-\int_{0}^{t} V^{\omega}\left(X_{s}\right) \mathrm{d} s} ; t<\tau_{\Lambda}\right] \mathrm{d} x,
\end{aligned}
$$

for any fixed $t>0$. Denoting by $L$ the Laplace transform of the measure $\ell$, we have

$$
L(t)=\lim _{\Lambda \nearrow \mathbb{R}^{d}} \mathbb{E}^{\mathbb{Q}} L_{\Lambda}^{\omega}(t), \quad t>0 .
$$

Let us note that from the $\mathbb{Z}^{d}$-stationarity of the potential we have that for any $\Lambda$ as above,

$$
L(t)=\frac{p_{t}(0,0)}{|\Lambda|} \int_{\Lambda} \mathbb{E}^{\mathbb{Q}} \mathbf{E}_{x, x}^{t}\left[\mathrm{e}^{-\int_{0}^{t} V^{\omega}\left(X_{s}\right) \mathrm{d} s}\right] \mathrm{d} x .
$$

In particular, for any $M \in \mathbb{Z}_{+}$,

$$
L(t)=\frac{p_{t}(0,0)}{M^{d}} \int_{[0, M)^{d}} \mathbb{E}^{\mathbb{Q}} \mathbf{E}_{x, x}^{t}\left[\mathrm{e}^{-\int_{0}^{t} V^{\omega}\left(X_{s}\right) \mathrm{d} s}\right] \mathrm{d} x .
$$


In the next two sections we will determine the rate of decay, as $t \rightarrow \infty$, of the Laplace transform $L(t)$ of the measure $\ell$.

\section{The UPPER BOUND FOR THE LAPLACE TRANSFORMS}

We start with the upper bounds, as they will determine the correct rate(s) in the asymptotics. We have more flexibility with lower bounds, thus the crucial step is to get a correct upper bound. To put oneself in a proper perspective, let us recall that for Lévy operators satisfying (B) perturbed by a Poissonian-type potential, the decay of $L(t)$ was of order $\mathrm{e}^{-C t^{\frac{d}{d+\alpha}}}$. We obtained a similar rate for the Anderson model for the fractional Laplacians, provided the distribution of the random variables $q_{\mathbf{i}}$ had an atom at zero. However, when the atom at zero is not present, then our earlier work [17] indicates that an extra multiplicative input is needed in the decay rate. As Theorems 3.1 and 4.2 show, this is indeed the case.

3.1. The rate function and the statement of the upper bound (Theorem 3.1). We start with the definition of the function $h(t)$ which will appear in the rate.

Let $\alpha \in(0,2]$ and $C_{1}>0$ be the scaling exponent and the constant from the assumption (B), and let $\kappa_{0}$ and $M_{0}$ be the parameters appearing in the assumptions (Q) and (W), respectively. Moreover, recall that by $\mu_{2}^{1}$ we have denoted the second eigenvalue of the operator $-\Delta^{1}$ (see Section [2.3). Denote

$$
D_{0}=\frac{1}{2} \frac{C_{1}\left(\mu_{2}^{1}\right)^{\frac{\alpha}{2}}\|W\|_{1}}{\|W\|_{1}^{2}+\left(2 M_{0}\right)^{d}\|W\|_{2}^{2}}>0 .
$$

As it will be seen below, in fact $D_{0}$ can be choosen to be an arbitrary constant for which

$$
\|W\|_{1}>\frac{D_{0}\left(2 M_{0}\right)^{d}\|W\|_{2}^{2}}{C_{1}\left(\mu_{2}^{1}\right)^{\frac{\alpha}{2}}-D_{0}\|W\|_{1}}>0,
$$

but for more clarity we prefer to keep $D_{0}$ fixed as in (3.1).

For $x>0$ let

$$
g(x)=\log \frac{1}{F_{q}\left(D_{0} / x\right)}=-\log \mathbb{Q}\left[q \leqslant D_{0} / x\right], \quad \text { and } \quad j(x)=x^{d+\alpha} g\left(x^{\alpha}\right) .
$$

Due to the assumption (Q) the function $j(x)$ is increasing, and continuous for $x \geqslant x_{0}:=$ $\left(D_{0} / \kappa_{0}\right)^{1 / \alpha}$. Therefore $j^{-1}(t)$ is well-defined for $t \geqslant t_{0}:=j\left(x_{0}\right)$. Let $x_{t}=j^{-1}(t), t \geqslant t_{0}$. Finally, denote

$$
h(t)=g\left(x_{t}^{\alpha}\right), \quad t \geqslant t_{0} .
$$

For later use, observe that $t$ and $x_{t}$ are related through the relation

$$
t=x_{t}^{d+\alpha} \log \left(\frac{1}{F_{q}\left(D_{0} / x_{t}^{\alpha}\right)}\right)=x_{t}^{d+\alpha} h(t), \quad t \geqslant t_{0} .
$$

Moreover, the function $t \mapsto x_{t}$ is increasing and since $\lim _{x \rightarrow \infty} j(x)=\infty$, we have $\lim _{t \rightarrow \infty} x_{t}=\infty$ as well. This implies that

$$
\lim _{t \rightarrow \infty} \frac{h(t)}{t}=0 .
$$

The $\operatorname{limit}_{\lim } \rightarrow \infty h(t)$ always exists and

$$
\lim _{t \rightarrow \infty} h(t)= \begin{cases}\infty & \text { if } \quad F_{q}(0)=0, \\ \log \frac{1}{F_{q}(0)} & \text { if } \quad F_{q}(0) \in(0,1) .\end{cases}
$$

In Section 6 we give examples of such functions $h$.

We are now ready to present the main theorem of this section. 
Theorem 3.1. Assume (B), (Q), and (W). Let $h$ be given by (3.4). Then there exists $C>0$ such that

$$
\limsup _{t \rightarrow \infty} \frac{\log L(t)}{t^{\frac{d}{d+\alpha}}(h(t))^{\frac{\alpha}{d+\alpha}}} \leqslant-C .
$$

In particular, when the distribution of $q$ has an atom at zero, i.e. $F_{q}(0)>0$, then

$$
\limsup _{t \rightarrow \infty} \frac{\log L(t)}{t^{\frac{d}{d+\alpha}}} \leqslant-C\left(\log \frac{1}{F_{q}(0)}\right)^{\frac{\alpha}{d+\alpha}} .
$$

The proof of the theorem is split into three parts which are presented in Sections 3.2, 3.3 and 3.4 below.

3.2. Preparatory steps in the proof of Theorem 3.1- the trace estimate. To begin the proof, we proceed as in [17, Proof of Theorem 4.1, the first page of Section 5.3]. As a corollary of [17. Lemma 5.1] we get that for any given $M \in \mathbb{Z}_{+}$and any $t>0$ we have the following relation between the exponential functionals of the subordinate Brownian motion $X=\left(X_{t}\right)_{t \geqslant 0}$ in $\mathbb{R}^{d}$ with the un-periodized and periodized potentials (cf. (2.11)-(2.12) ):

$$
\mathbb{E}^{\mathbb{Q}}\left[\mathrm{e}^{-\int_{0}^{t} V^{\omega}\left(X_{s}(w)\right) \mathrm{d} s}\right] \leqslant \mathbb{E}^{\mathbb{Q}}\left[\mathrm{e}^{-\int_{0}^{t} V_{M}^{\omega}\left(X_{s}(w)\right) \mathrm{d} s}\right],
$$

and further, invoking Lemma 2.4 ,

$$
L(t) \leqslant \frac{1}{M^{d}} \mathbb{E}^{\mathbb{Q}} \int_{\mathcal{T}_{M}} p_{t}^{M}(x, x) \mathbf{E}_{x, x}^{M, t}\left[\mathrm{e}^{-\int_{0}^{t} V_{M}^{\omega}\left(X_{s}^{M}\right) \mathrm{d} s}\right] \mathrm{d} x
$$

(recall that $X^{M}=\left(X_{s}^{M}\right)_{s \geqslant 0}$ is the subordinate Brownian motion on the torus $\mathcal{T}_{M}, p_{t}^{M}(\cdot, \cdot)$ are its transition densities, and $\mathbf{E}_{x, x}^{M, t}$ - its bridge measures).

The integral at the right-hand side of (3.10) is the trace of the random operator $P_{t}^{M, V_{M}^{\omega}}$ with integral kernel defined in (2.13). Consequently, for $t>1$,

$$
\begin{aligned}
L(t) & \leqslant \frac{1}{M^{d}} \mathbb{E}^{\mathbb{Q}} \operatorname{Tr} P_{t}^{M, V_{M}^{\omega}}=\frac{1}{M^{d}} \mathbb{E}^{\mathbb{Q}} \sum_{n=1}^{\infty} \mathrm{e}^{-t \lambda_{n}^{M, V_{M}^{\omega}}} \\
& \leqslant \frac{1}{M^{d}} \mathbb{E}^{\mathbb{Q}}\left[\mathrm{e}^{-(t-1) \lambda_{1}^{M, V_{M}^{\omega}}} \operatorname{Tr} P_{1}^{M, V_{M}^{\omega}}\right] \\
& \leqslant \mathbb{E}^{\mathbb{Q}}\left[\mathrm{e}^{-(t-1) \lambda_{1}^{M, V_{M}^{\omega}}}\right] \frac{1}{M^{d}} \int_{\mathcal{T}_{M}} p_{1}^{M}(x, x) \mathrm{d} x .
\end{aligned}
$$

From Lemma 2.3(2) there exists a constant $C>0$ independent of $M$ for which $p_{1}^{M}(x, x) \leqslant C$, $x \in \mathcal{T}_{M}$, so that we are led to the bound

$$
L(t) \leqslant C \mathbb{E}^{\mathbb{Q}}\left[\mathrm{e}^{-(t-1) \lambda_{1}^{M, V_{M}^{\omega}}}\right], \quad t>1 .
$$

3.3. Temple's inequality and the lower scaling of the ground state eigenvalue. In this section we find an appropriate lower estimate for the ground state eigenvalue $\lambda_{1}^{M, V_{M}^{\omega}}$ of the Schrödinger operator $H_{M}^{\omega}$. We will use the following inequality.

Proposition 3.2 (Temple's inequality, [33, Theorem XIII.5]). Suppose $H$ is a self-adjoint operator on a Hilbert space with inner product $\langle\cdot, \cdot\rangle$ such that $\lambda_{1}:=\inf \sigma(H)$ is an isolated eigenvalue and let $\mu \leqslant \inf \left(\sigma(H) \backslash\left\{\lambda_{1}\right\}\right)$. Then for any $\psi \in \mathcal{D}(H)$ which satisfies

$$
\langle\psi, H \psi\rangle<\mu \quad \text { and } \quad\|\psi\|=1
$$

the following bound holds:

$$
\lambda_{1} \geqslant\langle\psi, H \psi\rangle-\frac{\langle H \psi, H \psi\rangle-\langle\psi, H \psi\rangle^{2}}{\mu-\langle\psi, H \psi\rangle} .
$$


For given $M \in \mathbb{Z}_{+}$, consider truncated random variables

$$
\widetilde{q}_{\mathbf{i}}=q_{\mathbf{i}} \wedge \frac{D_{0}}{M^{\alpha}}, \quad \mathbf{i} \in \mathbb{Z}^{d}
$$

and random Schrödinger operators

$$
\widetilde{H}_{M}^{\omega}=\Phi\left(-\Delta^{M}\right)+\widetilde{V}_{M}^{\omega}
$$

where $\widetilde{V}_{M}^{\omega}$ is the Sznitman-periodization of

$$
\widetilde{V}^{\omega}(x)=\sum_{\mathbf{i} \in \mathbb{Z}^{d}} \widetilde{q}_{\mathbf{i}}(\omega) W(x-\mathbf{i}),
$$

cf. (2.11)-(2.12). We have a lemma.

Lemma 3.3. Let the assumptions (B) and (W) hold. Then for any $M \geqslant M_{0}$ and any constant $D_{0}>0$ satisfying (3.2) we have

$$
\lambda_{1}^{M, V_{M}^{\omega}} \geqslant \lambda_{1}^{M, \widetilde{V}_{M}^{\omega}} \geqslant \frac{1}{M^{d}}\left[\int_{\mathcal{T}_{M}} \widetilde{V}_{M}^{\omega}(x) \mathrm{d} x-\frac{\int_{\mathcal{T}_{M}}\left(\widetilde{V}_{M}^{\omega}(x)\right)^{2} \mathrm{~d} x}{\left(C_{1}\left(\mu_{2}^{1}\right)^{\frac{\alpha}{2}}-D_{0}\|W\|_{1}\right) \cdot M^{-\alpha}}\right] .
$$

Proof. Let $M \geqslant M_{0}$ be fixed. Since $\widetilde{V}_{M}^{\omega} \leqslant V_{M}^{\omega}$, the leftmost inequality in (3.16) is clear. We now apply Temple's inequality to the operator $\widetilde{H}_{M}^{\omega}$ acting in $L^{2}\left(\mathcal{T}_{M}\right)$, defined in (3.15), and $\mu=\lambda_{2}^{M}$. The spectrum of $\widetilde{H}_{M}^{\omega}$ is purely discrete and it is clear that

$$
\mu \leqslant \lambda_{2}^{M, \widetilde{V}_{M}^{\omega}}=\inf \left(\sigma\left(\widetilde{H}_{M}^{\omega}\right) \backslash\left\{\lambda_{1}^{M, \widetilde{V}_{M}^{\omega}}\right\}\right) .
$$

Let $\psi=\psi_{1}^{M} \equiv \frac{1}{M^{d / 2}}$. We have $\|\psi\|_{2}=1$ and, by (2.8)-(2.9),$\Phi\left(-\Delta^{M}\right) \psi=0$. Consequently,

$$
\left\langle\psi, \widetilde{H}_{M}^{\omega} \psi\right\rangle=\left\langle\psi, \Phi\left(-\Delta^{M}\right) \psi\right\rangle+\left\langle\psi, \widetilde{V}_{M}^{\omega} \psi\right\rangle=\left\langle\psi, \widetilde{V}_{M}^{\omega} \psi\right\rangle=\frac{1}{M^{d}} \int_{\mathcal{T}_{M}} \tilde{V}_{M}^{\omega}(x) \mathrm{d} x .
$$

By the definition of $\widetilde{V}_{M}^{\omega}$, we have

$$
\begin{aligned}
\int_{\mathcal{T}_{M}} \widetilde{V}_{M}^{\omega}(x) \mathrm{d} x & =\int_{\mathcal{T}_{M}} \sum_{\mathbf{i} \in[0, M)^{d}} \widetilde{q}_{\mathbf{i}}(\omega)\left(\sum_{\mathbf{i}^{\prime} \in \pi_{M}^{-1}(\mathbf{i})} W\left(x-\mathbf{i}^{\prime}\right)\right) \mathrm{d} x \\
& =\sum_{\mathbf{i} \in[0, M)^{d}} \widetilde{q}_{\mathbf{i}}(\omega)\left(\sum_{\mathbf{i}^{\prime} \in \pi_{M}^{-1}(\mathbf{i})} \int_{[0, M)^{d}-\mathbf{i}^{\prime}} W(x) \mathrm{d} x\right)=\|W\|_{1}\left(\sum_{\mathbf{i} \in[0, M)^{d}} \widetilde{q}_{\mathbf{i}}(\omega)\right) .
\end{aligned}
$$

Hence, by (3.14) and (3.1),

$$
\left\langle\psi, \widetilde{H}_{M}^{\omega} \psi\right\rangle \leqslant \frac{1}{M^{d}}\|W\|_{1} \cdot M^{d} \cdot \frac{D_{0}}{M^{\alpha}}=\frac{D_{0}\|W\|_{1}}{M^{\alpha}}<\frac{C_{1}\left(\mu_{2}^{1}\right)^{\frac{\alpha}{2}}}{M^{\alpha}} .
$$

On the other hand, from a combination of the lower bound in (1.7) and (2.7)-(2.8) it follows that

$$
\frac{C_{1}\left(\mu_{2}^{1}\right)^{\frac{\alpha}{2}}}{M^{\alpha}} \leqslant \Phi\left(M^{-2} \mu_{2}^{1}\right)=\Phi\left(\mu_{2}^{M}\right)=\lambda_{2}^{M},
$$

and therefore condition (3.12) is satisfied. For the ingredients of (3.13) we have:

$$
\begin{aligned}
\left\langle\psi, \widetilde{H}^{\omega} \psi\right\rangle & =\frac{1}{M^{d}} \int_{\mathcal{T}_{M}} \widetilde{V}_{M}^{\omega}(x) \mathrm{d} x, \\
\left\langle\widetilde{H}^{\omega} \psi, \widetilde{H}^{\omega} \psi\right\rangle & =\frac{1}{M^{d}} \int_{\mathcal{T}_{M}}\left(\widetilde{V}_{M}^{\omega}(x)\right)^{2} \mathrm{~d} x, \\
\mu-\left\langle\psi, \widetilde{H}^{\omega} \psi\right\rangle & =\lambda_{2}^{M}-\left\langle\psi, \widetilde{H}^{\omega} \psi\right\rangle \geqslant \frac{C_{1}\left(\mu_{2}^{1}\right)^{\frac{\alpha}{2}}}{M^{\alpha}}-\frac{D_{0}\|W\|_{1}}{M^{\alpha}}=\frac{C_{1}\left(\mu_{2}^{1}\right)^{\frac{\alpha}{2}}-D_{0}\|W\|_{1}}{M^{\alpha}} .
\end{aligned}
$$


Inserting these inside (3.13) we get

$$
\begin{aligned}
\lambda_{1}^{M, V_{M}^{\omega}} & \geqslant \lambda_{1}^{M, \widetilde{V}_{M}^{\omega}} \\
& \geqslant \frac{1}{M^{d}} \int_{\mathcal{T}_{M}} \widetilde{V}_{M}^{\omega}(x) \mathrm{d} x-\frac{\frac{1}{M^{d}} \int_{\mathcal{T}_{M}}\left(\widetilde{V}_{M}^{\omega}(x)\right)^{2} \mathrm{~d} x-\left(\frac{1}{M^{d}} \int_{\mathcal{T}_{M}} \widetilde{V}_{M}^{\omega}(x) \mathrm{d} x\right)^{2}}{\left(C_{1}\left(\mu_{2}^{1}\right)^{\frac{\alpha}{2}}-D_{0}\|W\|_{1}\right) M^{-\alpha}} \\
& \geqslant \frac{1}{M^{d}}\left[\int_{\mathcal{T}_{M}} \widetilde{V}_{M}^{\omega}(x) \mathrm{d} x-\frac{1}{\left(C_{1}\left(\mu_{2}^{1}\right)^{\frac{\alpha}{2}}-D_{0}\|W\|_{1}\right) M^{-\alpha}} \int_{\mathcal{T}_{M}}\left(\widetilde{V}_{M}^{\omega}(x)\right)^{2} \mathrm{~d} x\right],
\end{aligned}
$$

which is the desired statement.

This lemma will be useful when there is a lot of randomness in the picture, namely when the random variables $q_{\mathbf{i}}$ are bigger than $D_{0} / M^{\alpha}$ on a substantial part of sites in $\mathcal{T}_{M}$. To quantify this behavior, fix $\delta \in(0,1)$ (its actual value will be decided later) and consider the set

$$
\mathcal{A}_{M, \delta}=\left\{\omega: \#\left\{\mathbf{i} \in[0, M)^{d}: q_{\mathbf{i}}(\omega)>\frac{D_{0}}{M^{\alpha}}\right\} \geqslant \delta M^{d}\right\} .
$$

We have the following estimate.

Lemma 3.4. Let the assumptions (B) and (W) hold and let $\delta>0$ be fixed. Suppose $\omega \in \mathcal{A}_{M, \delta}$. Then for any $M \geqslant M_{0}$

$$
\lambda_{1}^{M, V_{M}^{\omega}} \geqslant D_{0} \cdot \delta\left[\|W\|_{1}-\frac{\left(2 M_{0}\right)^{d} D_{0}\|W\|_{2}^{2}}{C_{1}\left(\mu_{2}^{1}\right)^{\frac{\alpha}{2}}-D_{0}\|W\|_{1}}\right] \cdot \frac{1}{M^{\alpha}} .
$$

Proof. We have already shown in (3.17) that $\int_{\mathcal{T}_{M}} \widetilde{V}_{M}^{\omega}(x) \mathrm{d} x=\|W\|_{1}\left(\sum_{\mathbf{i} \in[0, M)^{d}} \widetilde{q}_{\mathbf{i}}(\omega)\right)$. Under present assumptions, we will also find a nice etimate on $\int_{\mathcal{T}_{M}}\left(\widetilde{V}_{M}^{\omega}(x)\right)^{2} \mathrm{~d} x$ and then we will apply Lemma 3.3. Observe that because of the assumption supp $W \subset\left[-M_{0}, M_{0}\right]^{d}$, in the sum defining $\widetilde{V}_{M}^{\omega}$,

$$
\widetilde{V}_{M}^{\omega}(x)=\sum_{\mathbf{i} \in[0, M)^{d}} \widetilde{q}_{\mathbf{i}}(\omega) \sum_{\mathbf{i}^{\prime} \in \pi_{M}^{-1}(\mathbf{i})} W\left(x-\mathbf{i}^{\prime}\right)
$$

there are at most $\left(2 M_{0}\right)^{d}$ nonzero terms and for every fixed $\mathbf{i} \in[0, M)^{d}$ the range of the summation $\pi_{M}^{-1}(\mathbf{i})$ in the inner sum contains at most one element. Consequently,

$$
\left(\widetilde{V}_{M}^{\omega}(x)\right)^{2} \leqslant\left(2 M_{0}\right)^{d} \sum_{\mathbf{i} \in[0, M)^{d}} \widetilde{q}_{\mathbf{i}}(\omega)^{2} \sum_{\mathbf{i}^{\prime} \in \pi_{M}^{-1}(\mathbf{i})} W\left(x-\mathbf{i}^{\prime}\right)^{2},
$$

and further, as in the proof of (3.17),

$$
\int_{\mathcal{T}_{M}}\left(\widetilde{V}_{M}^{\omega}(x)\right)^{2} \mathrm{~d} x \leqslant\left(2 M_{0}\right)^{d} \sum_{\mathbf{i} \in[0, M)^{d}} \widetilde{q}_{\mathbf{i}}^{2}(\omega)\|W\|_{2}^{2} .
$$

Inserting these estimates inside (3.16) we obtain:

$$
\begin{aligned}
\lambda_{1}^{M, V_{M}^{\omega}}\left(\mathcal{T}_{M}\right) & \geqslant \frac{1}{M^{d}}\left[\|W\|_{1} \sum_{\mathbf{i} \in[0, M)^{d}} \widetilde{q}_{\mathbf{i}}(\omega)-\frac{\left(2 M_{0}\right)^{d}}{\left(C_{1}\left(\mu_{2}^{1}\right)^{\frac{\alpha}{2}}-D_{0}\|W\|_{1}\right) M^{-\alpha}}\|W\|_{2}^{2} \sum_{\mathbf{i} \in[0, M)^{d}} \widetilde{q}_{\mathbf{i}}^{2}(\omega)\right] \\
& =\frac{1}{M^{d}} \sum_{\mathbf{i} \in[0, M)^{d}} \widetilde{q}_{\mathbf{i}}(\omega)\left[\|W\|_{1}-\frac{\left(2 M_{0}\right)^{d}\|W\|_{2}^{2} \sum_{\mathbf{i} \in[0, M)^{d}} \widetilde{q}_{\mathbf{i}}^{2}(\omega)}{\left(C_{1}\left(\mu_{2}^{1}\right)^{\frac{\alpha}{2}}-D_{0}\|W\|_{1}\right) M^{-\alpha}}\left(\sum_{\mathbf{i} \in[0, M)^{d}} \widetilde{q}_{\mathbf{i}}(\omega)\right)^{-1}\right] .
\end{aligned}
$$


Now: in the sum $\sum_{\mathbf{i} \in[0, M)^{d}} \widetilde{q}_{\mathbf{i}}(\omega)$ we keep only those i's for which $q_{\mathbf{i}}>\frac{D_{0}}{M^{\alpha}}$. Because of the assumption $\omega \in \mathcal{A}_{M, \delta}$, this leads to

$$
\sum_{\mathbf{i} \in[0, M)^{d}} \widetilde{q}_{\mathbf{i}}(\omega) \geqslant \frac{D_{0}}{M^{\alpha}} \cdot \delta M^{d},
$$

and for $\sum_{\mathbf{i} \in[0, M)^{d}} \widetilde{q}_{\mathbf{i}}^{2}(\omega)$ we write

$$
\sum_{\mathbf{i} \in[0, M)^{d}} \widetilde{q}_{\mathbf{i}}^{2}(\omega) \leqslant \frac{D_{0}}{M^{\alpha}} \sum_{\mathbf{i} \in[0, M)^{d}} \widetilde{q}_{\mathbf{i}}(\omega) .
$$

Finally,

$$
\lambda_{1}^{M, V_{M}^{\omega}} \geqslant \frac{D_{0} \delta}{M^{\alpha}}\left[\|W\|_{1}-\frac{\left(2 M_{0}\right)^{d} D_{0}\|W\|_{2}^{2}}{C_{1}\left(\mu_{2}^{1}\right)^{\frac{\alpha}{2}}-D_{0}\|W\|_{1}}\right] .
$$

3.4. Conclusion of the proof of Theorem 3.1 - derivation of the rate function. To conclude the proof we continue with the estimate of $L(t)$ from (3.11), splitting the $\mathbb{Q}$-integration into two parts: over $\mathcal{A}_{M, \delta}$ and over its complement.

For the integral over $\mathcal{A}_{M, \delta}$ we have the estimate from Lemma 3.4, and the integral over $\mathcal{A}_{M, \delta}^{c}$ is not bigger than $\mathbb{Q}\left[\mathcal{A}_{M, \delta}^{c}\right]$, whose probability can be estimated by the following Bernstein-type inequality on the binomial distribution. Its proof is an exercise from elementary probability, but we give here a short proof for the reader's convenience.

Lemma 3.5. Let $(\Omega, \mathcal{F}, \mathbb{P})$ be a given probability space and let $S_{n}: \Omega \rightarrow \mathbb{R}$ be a random variable with the binomial distribution $B(n, p), n \geqslant 1, p \in(0,1)$. Then, for any $p, \gamma \in(0,1)$ such that $\gamma>p$

$$
\mathbb{P}\left[S_{n} \geqslant \gamma n\right] \leqslant\left(\left(\frac{1-p}{1-\gamma}\right)^{1-\gamma}\left(\frac{p}{\gamma}\right)^{\gamma}\right)^{n} .
$$

Proof. For any given $t>0$, we have the following estimate, deduced from the Markov inequality:

$$
\mathbb{P}\left[S_{n} \geqslant \gamma n\right]=\mathbb{P}\left[\mathrm{e}^{t S_{n}} \geqslant \mathrm{e}^{t \gamma n}\right] \leqslant \frac{\mathbb{E} \mathrm{e}^{t S_{n}}}{\mathrm{e}^{t \gamma n}}=\left(\frac{p \mathrm{e}^{t}+1-p}{\mathrm{e}^{t \gamma}}\right)^{n} .
$$

The minimal value of the right-hand side is taken at $t_{\gamma}=\log \left(\frac{\gamma}{p} \frac{1-p}{1-\gamma}\right)$, which is positive for $\gamma>p$. This value is equal to $\left(\left(\frac{1-p}{1-\gamma}\right)^{1-\gamma}\left(\frac{p}{\gamma}\right)^{\gamma}\right)^{n}$, as claimed.

Proof of Theorem 3.1. For an arbitrary $\delta \in(0,1)$ we can write

$$
L(t) \leqslant C \mathbb{E}^{\mathbb{Q}}\left[\mathrm{e}^{-(t-1) \lambda_{1}^{M, V_{M}^{\omega}}} ; \mathcal{A}_{M, \delta}\right]+C \mathbb{Q}\left[\mathcal{A}_{M, \delta}^{c}\right], \quad t>1, \quad M \geqslant M_{0} .
$$

To make use of Lemma 3.5 observe

$$
\begin{aligned}
\mathcal{A}_{M, \delta}^{c} & =\left\{\omega: \#\left\{\mathbf{i} \in[0, M)^{d}: q_{\mathbf{i}}(\omega)>\frac{D_{0}}{M^{\alpha}}\right\}<\delta M^{d}\right\} \\
& =\left\{\omega: \#\left\{\mathbf{i} \in[0, M)^{d}: q_{\mathbf{i}}(\omega) \leqslant \frac{D_{0}}{M^{\alpha}}\right\} \geqslant(1-\delta) M^{d}\right\} .
\end{aligned}
$$

The events $A_{\mathbf{i}}=\left\{q_{\mathbf{i}} \leqslant \frac{D_{0}}{M^{\alpha}}\right\}$ are independent and have common probability $p_{M}=F_{q}\left(\frac{D_{0}}{M^{\alpha}}\right)$. Therefore we use the lemma with $n=M^{d}, p=p_{M}$ as above, and $\gamma=(1-\delta)$. We only need to make sure that $(1-\delta)>p_{M}$, i.e. $\delta<1-p_{M}$. As eventually we will let $M \rightarrow \infty$ and the 
distribution of the random variable $q$ is not concentrated at 0 , this will not be a problem. It then follows that for every $M \geqslant M_{0}$ and $\delta<1-p_{M}$ it holds

$$
\mathbb{Q}\left[\mathcal{A}_{M, \delta}^{c}\right] \leqslant\left[\left(\frac{1-p_{M}}{\delta}\right)^{\delta}\left(\frac{p_{M}}{1-\delta}\right)^{1-\delta}\right]^{M^{d}} \leqslant\left[\frac{1}{1-\delta}\left(\frac{1}{\delta}\right)^{\frac{\delta}{1-\delta}} \cdot p_{M}\right]^{(1-\delta) M^{d}} .
$$

Since $p_{M}=F_{q}\left(\frac{D_{0}}{M^{\alpha}}\right) \rightarrow F_{q}(0) \in[0,1)$ as $M \rightarrow \infty$ and $\frac{1}{1-\delta}\left(\frac{1}{\delta}\right)^{\frac{\delta}{1-\delta}} \searrow 1$ as $\delta \searrow 0$, we can easily find $M_{1} \geqslant M_{0}$ and $\delta_{0}<1-p_{M_{1}}$ such that for every $M \geqslant M_{1}$

$$
\frac{1}{1-\delta_{0}}\left(\frac{1}{\delta_{0}}\right)^{\frac{\delta_{0}}{1-\delta_{0}}} \cdot \sqrt{p_{M}} \leqslant 1
$$

(in particular, $\delta_{0}<1-p_{M}$, for $M \geqslant M_{1}$ as $p_{M}$ is nonincreasing in $M$ ). It gives

$$
\mathbb{Q}\left[\mathcal{A}_{M, \delta_{0}}^{c}\right] \leqslant p_{M}^{\left(1-\delta_{0}\right) M^{d} / 2}=\mathrm{e}^{-\frac{1-\delta_{0}}{2} M^{d} \log \left(1 / p_{M}\right)}, \quad M \geqslant M_{1} .
$$

Denote $c_{1}=\frac{D_{0} \delta_{0}}{2}\left[\|W\|_{1}-\frac{\left(2 M_{0}\right)^{d} D_{0}\|W\|_{2}^{2}}{\left(C_{1}\left(\mu_{2}^{1}\right)^{\frac{\alpha}{2}}-D_{0}\|W\|_{1}\right)}\right]$ and $c_{2}=\left(1-\delta_{0}\right) / 2$. We now insert the bounds (3.19) and (3.22) inside (3.21) and obtain that there exist $t_{0}>1$ such that for every $t \geqslant t_{0}$ and $M \geqslant M_{1}$ we have

$$
\begin{aligned}
& L(t) \leqslant C\left(\mathrm{e}^{-\frac{2 c_{1}(t-1)}{M^{\alpha}}}+\mathrm{e}^{-c_{2} M^{d} \log \frac{1}{F_{q}\left(D_{0} / M^{\alpha}\right)}}\right) \\
& \leqslant C\left(\mathrm{e}^{-\frac{c_{1} t}{(M-1)^{\alpha}}}+\mathrm{e}^{-c_{2} M^{d} \log \frac{1}{F_{q}\left(D_{0} / M^{\alpha}\right)}}\right) \leqslant C\left(\mathrm{e}^{-\frac{c_{3} t}{(M-1)^{\alpha}}}+\mathrm{e}^{-c_{3} M^{d} \log \frac{1}{F_{q}\left(D_{0} / M^{\alpha}\right)}}\right),
\end{aligned}
$$

with $c_{3}=\min \left(c_{1}, c_{2}\right)$.

So far, the bound we obtained was valid for any $M>M_{1}$. We will now make $M$ depend on $t$, in such a manner that $M \rightarrow \infty$ when $t \rightarrow \infty$. We will use the function $j(x)=x^{d+\alpha} \log \frac{1}{F_{q}\left(D_{0} / x^{\alpha}\right)}$ from (3.3). For $t \geqslant t_{0}$ (we may increase $t_{0}$ if necessary), the function $x_{t}:=j^{-1}(t)$ is well defined and obeys (3.5). Let us take

$$
M=\left\lfloor x_{t}\right\rfloor+1, \quad t \geqslant t_{0},
$$

i.e. $M$ is the unique integer satisfying $x_{t}-1<M-1 \leqslant x_{t}$. Clearly, there is $t_{1} \geqslant t_{0}$ such that for $t \geqslant t_{1}$ one has $M \geqslant M_{1}$. Consequently, by (3.5),

$$
(M-1)^{\alpha} \leqslant x_{t}^{\alpha}=\left(\frac{t}{\log \frac{1}{F_{q}\left(D_{0} / x_{t}^{\alpha}\right)}}\right)^{\frac{\alpha}{d+\alpha}}
$$

so that

$$
\frac{t}{(M-1)^{\alpha}} \geqslant t^{\frac{d}{d+\alpha}}\left(\log \frac{1}{F_{q}\left(D_{0} / x_{t}^{\alpha}\right)}\right)^{\frac{\alpha}{d+\alpha}}, \quad t \geqslant t_{1} .
$$

Next, as the function $x \mapsto x^{d} \log \frac{1}{F_{q}\left(D_{0} / x^{\alpha}\right)}$ is increasing and $x_{t}<M$, for the other exponent in (3.23) we have

$$
M^{d} \log \frac{1}{F_{q}\left(D_{0} / M^{\alpha}\right)} \geqslant x_{t}^{d} \log \frac{1}{F_{q}\left(D_{0} / x_{t}^{\alpha}\right)}=t^{\frac{d}{d+\alpha}}\left(\log \frac{1}{F_{q}\left(D_{0} / x_{t}^{\alpha}\right)}\right)^{\frac{\alpha}{d+\alpha}} .
$$

Consequently,

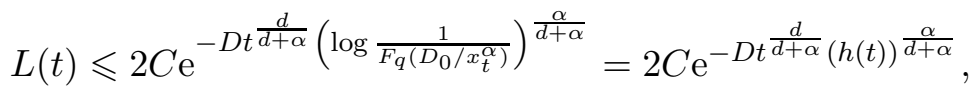

which yields (3.8). The second assertion (3.9) is an easy consequence of (3.8) and (3.7). 


\section{The LOWER BOUnd FOR THE LAPlaCe TRANSFORMS}

The matching lower bound will be obtained by restricting the integration in the integrals leading to $L(t)$ (see (2.17) ) to a smaller set, on which we will be able to control the expressions from below, and whose probability will be manageable. On this set we replace our random potential $V^{\omega}$ with deterministic potentials

$$
V_{\kappa}(x):=\kappa \sum_{\mathbf{i} \in[-M, 2 M)^{d}} W(x-\mathbf{i}), \quad \text { where } \kappa>0 \text { is some specially chosen parameter, }
$$

and then we estimate from the above the ground state eigenvalues of the Schrödinger operators

$$
\Phi(-\Delta)+V_{\kappa}
$$

constrained to large boxes in $\mathbb{R}^{d}$. Note that in this section we work with the operators $\Phi(-\Delta)$ and the subordinate Brownian motions in $\mathbb{R}^{d}$ and its sub-domains only, and we need not consider the operators and the processes on tori. We first recall necessary notation (cf. Section 2.6).

If $V \in \mathcal{K}_{\text {loc }}$ is a nonnegative potential and $\Lambda$ is a bounded domain in $\mathbb{R}^{d}$, then by $H_{\Lambda}$ we denote the Schrödinger operator $H=\Phi(-\Delta)+V$ constrained to $\Lambda$ (i.e. with Dirichlet conditions on $\Lambda^{c}$ in the non-local case and on $\partial \Lambda$ in the local case) and by $P_{t}^{V, \Lambda}=e^{-t H_{\Lambda}}$ the operators of its evolution semigroup. The ground state eigenvalue $\lambda_{1}^{V}(\Lambda)$ of $H_{\Lambda}$ can be represented through the variational formula

$$
\lambda_{1}^{V}(\Lambda)=\inf \left\{\mathcal{E}(\varphi, \varphi)+\int_{\Lambda} V(x) \varphi^{2}(x) d x: \varphi \in L^{2}(\Lambda),\|\varphi\|_{2}=1\right\} .
$$

This infimum is achieved for $\varphi_{1}^{V, \Lambda}$, the ground state eigenfunction of $H_{\Lambda}$. Below we also consider the case when $V \equiv 0$ for which we use simpler notation: $P_{t}^{\Lambda}, \lambda_{1}(\Lambda)$ and $\varphi_{1}^{\Lambda}$.

We start with an auxiliary lemma.

Lemma 4.1. Let $V: \mathbb{R}^{d} \rightarrow \mathbb{R}_{+}$be a potential that belongs to $\mathcal{K}_{\text {loc }} \cap L^{1}\left(\mathbb{R}^{d}\right)$. Then for any domain $\Lambda \subset \mathbb{R}^{d}$ we have

$$
\lambda_{1}^{V}(\Lambda) \leqslant \lambda_{1}(\Lambda)+\mathrm{e} p_{s}(0)\|V\|_{1}, \quad \text { with } s:=\frac{1}{\lambda_{1}(\Lambda)} .
$$

Proof. Choosing $s=\frac{1}{\lambda_{1}(\Lambda)}$ in the eigenequation $P_{s / 2}^{\Lambda} \varphi_{1}^{\Lambda}=\mathrm{e}^{-(s / 2) \lambda_{1}(\Lambda)} \varphi_{1}^{\Lambda}$, we get

$$
\begin{aligned}
\varphi_{1}^{\Lambda}(x) & =\sqrt{\mathrm{e}} \int_{\Lambda} \varphi_{1}^{\Lambda}(y) p_{s / 2}^{\Lambda}(x, y) \mathrm{d} y \leqslant \sqrt{\mathrm{e}}\left(\int_{\Lambda}\left(\varphi_{1}^{\Lambda}(y)\right)^{2} \mathrm{~d} y\right)^{1 / 2}\left(\int_{\Lambda}\left(p_{s / 2}^{\Lambda}(x, y)\right)^{2} \mathrm{~d} y\right)^{1 / 2} \\
& \leqslant \sqrt{\mathrm{e}}\left\|\varphi_{1}^{\Lambda}\right\|_{2}\left(p_{s}(x, x)\right)^{1 / 2}=\sqrt{\mathrm{e} p_{s}(0)} .
\end{aligned}
$$

By (4.1) we then obtain

$$
\lambda_{1}^{V}(\Lambda) \leqslant \mathcal{E}\left(\varphi_{1}^{\Lambda}, \varphi_{1}^{\Lambda}\right)+\int_{\Lambda} V(x)\left(\varphi_{1}^{\Lambda}(x)\right)^{2} \mathrm{~d} x \leqslant \lambda_{1}(\Lambda)+\mathrm{e} p_{s}(0)\|V\|_{1} .
$$

The following is the main theorem of this section.

Theorem 4.2. Assume (B), (Q), and (W). Let $h$ be given by (3.4). Then there exists $C>0$ such that

$$
\liminf _{t \rightarrow \infty} \frac{\log L(t)}{t^{\frac{d}{d+\alpha}}(h(t))^{\frac{\alpha}{d+\alpha}}} \geqslant-C .
$$

In particular, when the distribution of $q$ has an atom at zero, i.e. $F_{q}(0)>0$, then

$$
\liminf _{t \rightarrow \infty} \frac{\log L(t)}{t^{\frac{d}{d+\alpha}}} \geqslant-C\left(\log \frac{1}{F_{q}(0)}\right)^{\frac{\alpha}{d+\alpha}}
$$


Proof. Let $\Lambda_{M}:=[0, M)^{d}, M \in \mathbb{Z}_{+}$. Recall that by (2.17), for any $t>0$, we have

$$
\begin{aligned}
L(t) & =\frac{p_{t}(0,0)}{M^{d}} \int_{\Lambda_{M}} \mathbf{E}_{x, x}^{t} \mathbb{E}^{\mathbb{Q}}\left[\mathrm{e}^{-\int_{0}^{t} V^{\omega}\left(X_{s}\right) \mathrm{d} s}\right] \mathrm{d} x \\
& =\frac{1}{M^{d}} \int_{\Lambda_{M}} p_{t}(x, x) \mathbf{E}_{x, x}^{t} \mathbb{E}^{\mathbb{Q}}\left[\mathrm{e}^{-\int_{0}^{t} V^{\omega}\left(X_{s}\right) \mathrm{d} s}\right] \mathrm{d} x, \quad M \in \mathbb{Z}_{+} .
\end{aligned}
$$

For given $M \geqslant M_{0}$ (recall that $M_{0}$ comes from the Assumption (W)) and $\kappa>0$ let

$$
\mathcal{A}_{\kappa}^{M}=\left\{\omega: \forall \mathbf{i} \in[-M, 2 M)^{d} \text { we have } q_{\mathbf{i}}(\omega) \leqslant \kappa\right\}
$$

and restrict the inner expectations in (4.5) to the set $\mathcal{A}_{\kappa}^{M} \cap\left\{t<\tau_{\Lambda_{M}}\right\}$. For later use, observe that

$$
\mathbb{Q}\left[\mathcal{A}_{\kappa}^{M}\right]=\left(F_{q}(\kappa)\right)^{(3 M)^{d}}=\mathrm{e}^{-(3 M)^{d} \log \frac{1}{F_{q}(\kappa)}} .
$$

On the set $\left\{t<\tau_{\Lambda_{M}}\right\}$ we have

$$
V^{\omega}\left(X_{s}\right)=\sum_{\mathbf{i} \in[-M, 2 M)^{d}} q_{\mathbf{i}}(\omega) W\left(X_{s}-\mathbf{i}\right), \quad s \leqslant t .
$$

This is due to the fact that when $x-y \notin[-M, M]^{d}$, then $W(x-y)=0$. Next, for $\omega \in \mathcal{A}_{\kappa}^{M}$, all the $q_{\mathbf{i}}$ 's in the first sum above are not bigger than $\kappa$. It follows that

$$
\begin{aligned}
\mathbb{E}^{\mathbb{Q}}\left[\mathrm{e}^{-\int_{0}^{t} V^{\omega}\left(X_{s}\right) \mathrm{d} s} ; \mathcal{A}_{\kappa}^{M}\right] & \geqslant \mathbb{E}^{\mathbb{Q}}\left[\mathrm{e}^{-\int_{0}^{t} \kappa \sum_{i \in[-M, 2 M)^{d}} W\left(X_{s}-\mathbf{i}\right) \mathrm{d} s} ; \mathcal{A}_{\kappa}^{M}\right] \\
& \geqslant \mathrm{e}^{-\int_{0}^{t} \kappa \sum_{i \in[-M, 2 M)^{d}} W\left(X_{s}-\mathbf{i}\right) \mathrm{d} s} \mathbb{Q}\left[\mathcal{A}_{\kappa}^{M}\right] .
\end{aligned}
$$

We see that for $M \geqslant M_{0}$

$$
L(t) \geqslant \frac{1}{M^{d}} \mathbb{Q}\left[\mathcal{A}_{\kappa}^{M}\right] \int_{\Lambda_{M}} p(t, x, x) \mathbf{E}_{x, x}^{t}\left[\mathrm{e}^{-\kappa \int_{0}^{t} \sum_{\mathbf{i} \in[-M, 2 M)^{d}} W\left(X_{s}-\mathbf{i}\right) \mathrm{d} s} ; t<\tau_{\Lambda_{M}}\right] \mathrm{d} x .
$$

In the integral over $\Lambda_{M}$ we recognize the trace of the operator $P_{t}^{V_{\kappa}, \Lambda_{M}}$ (cf. (2.14) $)$ on $L^{2}\left(\Lambda_{M}\right)$, corresponding to the potential

$$
V_{\kappa}(x)=\kappa \sum_{\mathbf{i} \in[-M, 2 M)^{d}} W(x-\mathbf{i}) .
$$

Therefore this integral is not bigger than the principal eigenvalue of the operator $P_{t}^{V_{\kappa}, \Lambda_{M}}$, which in turn can be estimated by Lemma 4.1 .

$$
\operatorname{Tr} P_{t}^{V_{\kappa}, \Lambda_{M}} \geqslant \mathrm{e}^{-t \lambda_{1}^{V_{\kappa}}\left(\Lambda_{M}\right)} \geqslant \mathrm{e}^{-t\left(\lambda_{1}\left(\Lambda_{M}\right)+\mathrm{e} p_{s}(0)\left\|V_{\kappa}\right\|_{1}\right)}, \quad \text { with } s=\frac{1}{\lambda_{1}\left(\Lambda_{M}\right)} .
$$

It remains to estimate the $L^{1}$-norm of $V_{\kappa}$. We have:

$$
\begin{aligned}
\left\|V_{\kappa}\right\|_{1} & =\kappa \int_{\mathbb{R}^{d}} \sum_{\mathbf{i} \in[-M, 2 M)^{d}} W(x-\mathbf{i}) \mathrm{d} x=\kappa \sum_{\mathbf{i} \in[-M, 2 M)^{d}} \int_{\mathbb{R}^{d}} W(x) \mathrm{d} x \\
& =\kappa(3 M)^{d}\|W\|_{1} .
\end{aligned}
$$

From the estimates (4.6), (4.8), and (4.9) we then obtain that for $M \geqslant M_{0}$ and $t>0$

$$
L(t) \geqslant \frac{1}{M^{d}} \mathrm{e}^{-(3 M)^{d} \log \frac{1}{F_{q}(\kappa)}} \mathrm{e}^{-t\left(\lambda_{1}\left(\Lambda_{M}\right)+\mathrm{e} \kappa p_{s}(0)(3 M)^{d}\|W\|_{1}\right)}, \quad \text { with } s=\frac{1}{\lambda_{1}\left(\Lambda_{M}\right)} .
$$

Furthermore, it follows from [7, Theorem 3.4] that

$$
\lambda_{1}\left(\Lambda_{M}\right) \leqslant \Phi\left(\mu_{1}\left(\Lambda_{M}\right)\right),
$$

where $\mu_{1}\left(\Lambda_{M}\right)$ is the ground state eigenvalue of the Laplace operator $-\Delta$ on $\Lambda_{M}$ with Dirichlet boundary conditions. Since $\mu_{1}\left(\Lambda_{M}\right)=M^{-2} \mu_{1}\left(\Lambda_{1}\right)$, by the upper bound in the assumption (B) we obtain that there exist $M_{1} \geqslant M_{0}$ and a constant $c_{1}>0$ such that

$$
\lambda_{1}\left(\Lambda_{M}\right) \leqslant \frac{c_{1}}{M^{\alpha}}, \quad M \geqslant M_{1} .
$$


In particular, by (2.3), there is a constant $c_{2}>0$, for which we get

$$
\left.p_{s}(0)\right|_{s=\frac{1}{\lambda_{1}\left(\Lambda_{M}\right)}} \leqslant \frac{c_{2}}{M^{d}}, \quad M \geqslant M_{1} .
$$

Consequently, by choosing $\kappa=\frac{D_{0}}{M^{\alpha}}$, where $D_{0}$ comes from (3.1) (same as in the proof of the upper bound), we obtain

$$
L(t) \geqslant \frac{1}{M^{d}} \mathrm{e}^{-c_{3}\left(\frac{t}{(M+1)^{\alpha}}+M^{d}\left(\frac{M}{M+1}\right)^{\alpha} \log \frac{1}{F_{q}\left(D_{0} / M^{\alpha}\right)}\right)}, \quad M \geqslant M_{1},
$$

for some constant $c_{3}>0$. The exponent can be written as

$$
-\frac{c_{3}}{(M+1)^{\alpha}}\left(t+M^{d+\alpha} \log \frac{1}{F_{q}\left(D_{0} / M^{\alpha}\right)}\right) .
$$

Again, assume $t \geqslant t_{0}$, let $x_{t}=j^{-1}(t)$ (for a definition of the function $j$ and $t_{0}$ see the formula (3.3) and the two sentences following it), and choose $M=\left\lfloor x_{t}\right\rfloor$. It is the unique integer for which

$$
M \leqslant x_{t}<M+1, \quad \text { i.e. } \quad j(M) \leqslant t<j(M+1) .
$$

Condition $j(M) \leqslant t$ reads $M^{d+\alpha} \log \frac{1}{F_{q}\left(D_{0} / M^{\alpha}\right)} \leqslant t$. Moreover, by (3.5),

$$
\frac{t}{(M+1)^{\alpha}} \leqslant \frac{t}{x_{t}^{\alpha}}=t^{\frac{d}{d+\alpha}}\left(\log \frac{1}{F_{q}\left(D_{0} / x_{t}^{\alpha}\right)}\right)^{\frac{\alpha}{d+\alpha}}=t^{\frac{d}{d+\alpha}}(h(t))^{\frac{\alpha}{d+\alpha}} .
$$

Consequently, there is a number $t_{1} \geqslant t_{0}$ such that for $t \geqslant t_{1}$

$$
L(t) \geqslant \frac{1}{M^{d}} \mathrm{e}^{-2 c_{3} t^{\frac{d}{d+\alpha}}(h(t))^{\frac{\alpha}{d+\alpha}}},
$$

with $M$ chosen as above, i.e.

$$
\frac{\log L(t)}{t^{\frac{d}{d+\alpha}}(h(t))^{\frac{\alpha}{d+\alpha}}} \geqslant-2 c_{3}-d \frac{\log \left\lfloor x_{t}\right\rfloor}{t^{\frac{d}{d+\alpha}}(h(t))^{\frac{\alpha}{d+\alpha}}} .
$$

To conclude, we only need to verify that

$$
\lim _{t \rightarrow \infty} \frac{\log \left\lfloor x_{t}\right\rfloor}{t^{\frac{d}{d+\alpha}}(h(t))^{\frac{\alpha}{d+\alpha}}}=0 .
$$

This is clear as, by (3.5), $t=x_{t}^{d+\alpha} h(t)$, and further $(d+\alpha) \log x_{t}+\log h(t)=\log t$, so that

$$
\frac{\log x_{t}}{t^{\frac{d}{d+\alpha}}(h(t))^{\frac{\alpha}{d+\alpha}}}=\frac{1}{(d+\alpha)} \frac{\log t-\log h(t)}{t^{\frac{d}{d+\alpha}}(h(t))^{\frac{\alpha}{d+\alpha}}} \rightarrow 0, \quad \text { as } t \rightarrow \infty,
$$

because $\lim _{t \rightarrow \infty} h(t)$ always exists and is strictly positive (possibly infinite, see (3.7)). Denoting $C=2 c_{3}$ and taking liminf in (4.11), we obtain (4.3). The second assertion (4.4) is again a direct consequence of (3.7).

\section{TAUbeRIAN THEOREMS AND THE ASYMPtotics OF THE IDS}

We will now transform the estimates for the Laplace transform $L(t)$ of the IDS obtained in Sections 3 and 4 into statements concerning the IDS itself. When $\log L(t) \asymp-t^{\gamma}$ as $t \rightarrow \infty$, with $\gamma \in(0,1)$, then one just uses the exponential Tauberian theorem [11, Theorem 2.1], to get $\log \ell(\lambda) \asymp-\lambda^{-\gamma /(1-\gamma)}, \lambda \searrow 0$, as it was done previously in [29, 32, 38, 31, 16, 17]. However, the rate we identified in Theorems 3.1, 4.2 is more general (a correction term is present) and the Tauberian theorems existing in the literature are not sufficient to deal with it. Therefore we first need to state and prove a version of exponential Tauberian theorem which can be applied in our situation. 
5.1. Tauberian theorem. The setting is as follows. Let $\rho(\mathrm{d} x)$ be a $\sigma$-finite Borel measure on $[0, \infty)$ and let $L(t):=\int_{[0, \infty)} e^{-t x} \rho(\mathrm{d} x)$ be its Laplace transform. We assume that $L(t)<\infty$ for every $t>0$. We will use the same letter $\rho$ for the cumulative distribution function of the measure $\rho$, i.e. $\rho(x)=\rho([0, x]), x \geqslant 0$. Moreover, let $g:(0, \infty) \rightarrow(0, \infty)$ be a nondecreasing function, continuous on $\left[x_{0}, \infty\right), x_{0} \geqslant 0$. Let $\alpha, d>0$ be two given numbers. For $t \geqslant t_{0}:=x_{0}^{d+\alpha} g\left(x_{0}^{\alpha}\right)$ there is a unique number $x_{t}$ such that $t=x_{t}^{d+\alpha} g\left(x_{t}^{\alpha}\right)$. Since $x^{d+\alpha} g\left(x^{\alpha}\right) \rightarrow \infty$ as $x \rightarrow \infty$, we also have $x_{t} \rightarrow \infty$ as $t \rightarrow \infty$. Finally, let $h(t)=g\left(x_{t}^{\alpha}\right), t \geqslant t_{0}$. Clearly, $\lim _{t \rightarrow \infty} h(t)$ exists and $\lim _{t \rightarrow \infty} h(t) \in(0, \infty]$. In particular, $t^{\frac{d}{d+\alpha}}(h(t))^{\frac{\alpha}{d+\alpha}} \rightarrow \infty$ as $t \rightarrow \infty$.

Theorem 5.1. Using the notation introduced above we have the following.

(i) If

$$
\liminf _{t \rightarrow \infty} \frac{\log L(t)}{t^{\frac{d}{d+\alpha}}(h(t))^{\frac{\alpha}{d+\alpha}}} \geqslant-A_{1},
$$

with certain constant $A_{1} \in(0, \infty)$, then for any $B_{1}>A_{1}$ we have

$$
\liminf _{x \rightarrow 0^{+}} \frac{x^{d / \alpha}}{g\left(B_{1} / x\right)} \log \rho(x) \geqslant-A_{1} B_{1}^{d / \alpha} \text {. }
$$

(ii) If

$$
\limsup _{t \rightarrow \infty} \frac{\log L(t)}{t^{\frac{d}{d+\alpha}}(h(t))^{\frac{\alpha}{d+\alpha}}} \leqslant-A_{2},
$$

with certain constant $A_{2} \in(0, \infty)$, then for any $B_{2}<A_{2}$

$$
\limsup _{x \rightarrow 0^{+}} \frac{x^{d / \alpha}}{g\left(B_{2} / x\right)} \log \rho(x) \leqslant-\left(A_{2}-B_{2}\right) B_{2}^{d / \alpha} .
$$

Proof. (i) Assume that (5.1) holds. To shorten the notation, denote $\gamma=\frac{d}{d+\alpha}$. Then the rate in the denominator of (5.1) (and of (5.3) ) is equal to $t^{\gamma}(h(t))^{1-\gamma}$. Let

$$
\widetilde{L}(t):=\int_{0}^{\infty} \mathrm{e}^{-t x} \rho(x) \mathrm{d} x=\frac{1}{t} L(t)
$$

(the last identity is obtained via integration by parts). It follows that (5.1) is satisfied for $\widetilde{L}(t)$ as well. Let $\epsilon>0$ be given. Then there is $t_{\epsilon}>0$ such that for $t>t_{\epsilon}$ one has

$$
\widetilde{L}(t) \geqslant \mathrm{e}^{-\left(A_{1}+\epsilon\right) t^{\gamma}(h(t))^{1-\gamma}} .
$$

Next, take $B_{1}>A_{1}$ and write

$$
\int_{0}^{B_{1} t^{\gamma-1}(h(t))^{1-\gamma}} \mathrm{e}^{-t x} \rho(x) \mathrm{d} x=\widetilde{L}(t)-\int_{B_{1} t^{-1}(h(t))^{1-\gamma}}^{\infty} \mathrm{e}^{-t x} \rho(x) \mathrm{d} x .
$$

The left-hand side of (5.6) is not bigger than

$$
\rho\left(B_{1} t^{\gamma-1}(h(t))^{1-\gamma}\right) \int_{0}^{B_{1} t^{\gamma-1}(h(t))^{1-\gamma}} \mathrm{e}^{-t x} \mathrm{~d} x=\rho\left(B_{1} t^{\gamma-1}(h(t))^{1-\gamma}\right) \frac{1-\mathrm{e}^{-B_{1} t^{\gamma}(h(t))^{1-\gamma}}}{t} .
$$

Moreover,

$$
\begin{aligned}
\int_{B_{1} t^{\gamma-1}(h(t))^{1-\gamma}}^{\infty} \mathrm{e}^{-t x} \rho(x) \mathrm{d} x & =\int_{B_{1} t^{\gamma-1}(h(t))^{1-\gamma}}^{\infty} \mathrm{e}^{-t \epsilon x} \mathrm{e}^{-t x(1-\epsilon)} \rho(x) \mathrm{d} x \\
& \leqslant \mathrm{e}^{-(1-\epsilon) B_{1} t^{\gamma}(h(t))^{1-\gamma}} \widetilde{L}(\epsilon t)
\end{aligned}
$$


which in the light of (5.5) yield

$$
\begin{aligned}
\rho\left(B_{1} t^{\gamma-1}(h(t))^{1-\gamma}\right) \frac{1-\mathrm{e}^{-B_{1} t^{\gamma}(h(t))^{1-\gamma}}}{t} & \geqslant \mathrm{e}^{-\left(A_{1}+\epsilon\right) t^{\gamma}(h(t))^{1-\gamma}}-\widetilde{L}(\epsilon t) \mathrm{e}^{-B_{1}(1-\epsilon) t^{\gamma} h(t)^{1-\gamma}} \\
& =\mathrm{e}^{-\left(A_{1}+\epsilon\right) t^{\gamma}(h(t))^{1-\gamma}}\left(1-\widetilde{L}(\epsilon t) \mathrm{e}^{t^{\gamma} h(t)^{1-\gamma}\left[-B_{1}(1-\epsilon)+\left(A_{1}+\epsilon\right)\right]}\right) \\
& =\mathrm{e}^{-\left(A_{1}+\epsilon\right) t^{\gamma}(h(t))^{1-\gamma}}(1+o(1)), \quad t \rightarrow \infty,
\end{aligned}
$$

provided $\epsilon<\frac{B_{1}-A_{1}}{B_{1}+1}$. It follows

$$
\begin{aligned}
\log \rho\left(B_{1} t^{\gamma-1}(h(t))^{1-\gamma}\right) \geqslant & -\left(A_{1}+\epsilon\right) t^{\gamma}(h(t))^{1-\gamma} \\
& +\log (1+o(1))+\log t-\log \left(1-\mathrm{e}^{B_{1} t^{\gamma}(h(t))^{1-\gamma}}\right)
\end{aligned}
$$

As mentioned above, $t^{\gamma}(h(t))^{1-\gamma} \rightarrow \infty$ as $t \rightarrow \infty$, and consequently, for any $B_{1}>A_{1}$ and $\epsilon$ sufficiently small,

$$
\liminf _{t \rightarrow \infty} \frac{\log \rho\left(B_{1} t^{\gamma-1}(h(t))^{1-\gamma}\right)}{t^{\gamma}(h(t))^{1-\gamma}} \geqslant-\left(A_{1}+\epsilon\right) .
$$

Now, the number $\epsilon$ on the right-hand side can be sent to zero and eliminated.

To conclude the proof of part (i), substitute $x=B_{1} t^{\gamma-1}(h(t))^{1-\gamma}$. We need to write $t^{\gamma}(h(t))^{1-\gamma}$ as a function of $x$. Recall that

$$
t=x_{t}^{d+\alpha} g\left(x_{t}^{\alpha}\right)=x_{t}^{d+\alpha} h(t), \quad \text { i.e. } \quad \frac{h(t)}{t}=x_{t}^{-(d+\alpha)} .
$$

It means

$$
x=B_{1}\left(\frac{h(t)}{t}\right)^{1-\gamma}=\frac{B_{1}}{x_{t}^{\alpha}} .
$$

Consequently,

$$
t^{\gamma}(h(t))^{1-\gamma}=\frac{t x}{B_{1}}=\frac{t}{x_{t}^{\alpha}}=x_{t}^{d} g\left(x_{t}^{\alpha}\right)=\frac{B_{1}^{d / \alpha}}{x^{d / \alpha}} g\left(\frac{B_{1}}{x}\right) .
$$

Assertion (5.2) follows.

(ii) Assume now that (5.3) holds. By an argument identical as above, (5.3) is satisfied for $\widetilde{L}(t)$. Then for any $\epsilon>0$ there is $t_{\epsilon}>0$ such that for $t>t_{\epsilon}$

$$
\widetilde{L}(t) \leqslant \mathrm{e}^{-\left(A_{2}-\epsilon\right) t^{\gamma}(h(t))^{1-\gamma}},
$$

and on the other hand, with any $B_{2}>0$,

$$
\widetilde{L}(t) \geqslant \int_{B_{2} t^{\gamma-1}(h(t))^{1-\gamma}}^{\infty} \mathrm{e}^{-t x} \rho(x) \mathrm{d} x \geqslant \rho\left(B_{2} t^{\gamma-1}(h(t))^{1-\gamma}\right) \cdot \frac{1}{t} \mathrm{e}^{-B_{2} t^{\gamma}(h(t))^{1-\gamma}} .
$$

Using both these inequalities, taking logarithm and rearranging we arrive at

$$
\frac{\log \rho\left(B_{2} t^{\gamma-1}(h(t))^{1-\gamma}\right)}{t^{\gamma}(h(t))^{1-\gamma}} \leqslant \frac{\log t}{t^{\gamma}(h(t))^{1-\gamma}}-\left(A_{2}-\epsilon-B_{2}\right), \quad t>t_{\epsilon}
$$

and further

$$
\limsup _{t \rightarrow \infty} \frac{\log \rho\left(B_{2} t^{\gamma-1}(h(t))^{1-\gamma}\right)}{t^{\gamma}(h(t))^{1-\gamma}} \leqslant-\left(A_{2}-\epsilon-B_{2}\right),
$$

which yields a viable result for $B_{2}<A_{2}$ (again, $\epsilon$ can be eliminated).

To conclude the proof, similarly as in part (i), we substitute $x=B_{2} t^{\gamma-1}(h(t))^{1-\gamma}$, getting

$$
t^{\gamma}(h(t))^{1-\gamma}=\frac{t x}{B_{2}}=\frac{t}{x_{t}^{\alpha}}=x_{t}^{d} g\left(x_{t}^{\alpha}\right)=\frac{B_{2}^{d / \alpha}}{x^{d / \alpha}} g\left(\frac{B_{2}}{x}\right) .
$$

This gives (5.4) and completes the proof. 
Remark 5.2. In the exponential Tauberian theorems without a correction term (cf. [11, Theorem 2.1]), one was able to handle constants $B_{1}$ and $B_{2}$. Now we do not know, in general, what the function $g$ looks like and how it behaves asymptotically. We will be able to get rid of those constants in particular cases only.

5.2. Asymptotics of the IDS. Finally, applying the Tauberian theorem from the previous section, we give the formal proof of Lifshitz tail asymptotics of the IDS in Theorem 1.1 stated in the Introduction.

Proof of Theorem [1.1. The result follows directly from Theorems 3.1, 4.2 and 5.1 with $g(x)=$ $\log \frac{1}{F_{q}\left(D_{0} / x\right)}, j(x)=x^{d+\alpha} g\left(x^{\alpha}\right), x_{t}=j^{-1}(t)$, and $h(t)=g\left(x_{t}^{\alpha}\right)$ as in Section 3.1.

We complement our presentation with less precise statements (the 'loglog' regime), matching the usual statement of the Lifshitz tail sometimes found in the literature.

First we show that the behavior of $\log g(x), \log x_{t}$ and $\log h(t)$ (see Section 3.1) at infinity are closely related.

Lemma 5.3. Let $g(x)=\log \frac{1}{F_{q}\left(D_{0} / x\right)}, j(x)=x^{d+\alpha} g\left(x^{\alpha}\right), x_{t}=j^{-1}(t)$, and $h(t)=g\left(x_{t}^{\alpha}\right)$. The following three conditions are equivalent:

(i) $\lim _{x \rightarrow \infty} \frac{\log g(x)}{\log x}$ exists and is equal to $a \in[0, \infty]$;

(ii) $\lim _{t \rightarrow \infty} \frac{\log x_{t}}{\log t}$ exists and is equal to $b \in[0,1 /(d+\alpha)]$;

(iii) $\lim _{t \rightarrow \infty} \frac{\log h(t)}{\log t}$ exists and is equal to $c \in[0,1]$.

Numbers $a, b$ and $c$ are related through

$$
b=\frac{1}{d+(a+1) \alpha} \quad \text { and } \quad c=1-(d+\alpha) b=1-\frac{d+\alpha}{d+(a+1) \alpha}
$$

(here we use the standard convention $1 /+\infty=0$ and $1 / 0^{+}=+\infty$ ).

Proof. Recall that by (3.5)

$$
t=x_{t}^{d+\alpha} g\left(x_{t}^{\alpha}\right)=x_{t}^{d+\alpha} h(t), \quad t \geqslant t_{0} .
$$

In particular,

$$
\log h(t)=\log t-(d+\alpha) \log x_{t}, \quad t \geqslant t_{0},
$$

and

$$
\frac{\log g\left(x_{t}^{\alpha}\right)}{\log x_{t}^{\alpha}}=\frac{\log h(t)}{\log x_{t}^{\alpha}}=\frac{1}{\alpha} \frac{\log t}{\log x_{t}}-\frac{d+\alpha}{\alpha}, \quad t \geqslant t_{0} .
$$

It follows directly from (5.10) that (ii) and (iii) are equivalent and $c=1-(d+\alpha) b$ (or, equivalently, $b=(1-c) /(d+\alpha))$. Moreover, by (5.11) and by the fact that $x_{t} \rightarrow \infty$ as $t \rightarrow \infty$, we see that (i) implies (ii) and then $b=1 /(d+(a+1) \alpha)$ (In particular, (i) gives (iii) with $c=1-(d+\alpha) /(d+(a+1) \alpha)$.) The converse implication (ii) $\Rightarrow$ (i) also follows from (5.11) by the fact that $\left[t_{0}, \infty\right) \ni t \mapsto x_{t}^{\alpha}$ is a continuous and increasing function onto $\left[x_{t_{0}}^{\alpha}, \infty\right)$.

Corollary 5.4. Suppose that (B), (Q), and (W) hold true. If $\lim _{x \rightarrow \infty} \frac{\log g(x)}{\log x}$ exists, then

$$
\lim _{x \rightarrow 0^{+}} \frac{\log |\log \ell(x)|}{\log x}=-\frac{d}{\alpha}-\lim _{x \rightarrow \infty} \frac{\log g(x)}{\log x}
$$

and

$$
\lim _{t \rightarrow \infty} \frac{\log |\log L(t)|}{\log t}=1-\frac{\alpha}{d+\left(1+\lim _{x \rightarrow \infty} \frac{\log g(x)}{\log x}\right) \alpha} .
$$

In particular, when $g(x)$ is of order lower than power-law (i.e. $\lim _{x \rightarrow \infty} \frac{\log g(x)}{\log x}=0$ ), then

$$
\lim _{x \rightarrow 0^{+}} \frac{\log |\log \ell(x)|}{\log x}=-\frac{d}{\alpha}
$$


and

$$
\lim _{t \rightarrow \infty} \frac{\log |\log L(t)|}{\log t}=\frac{d}{d+\alpha} .
$$

Proof. The assertion for the IDS follows directly from the estimates in Theorem 1.1 and the definition of limsup and liminf. For a proof of the second assertion, for $L(t)$, observe that by Theorems 3.1 and 4.2 , the definition of limsup and liminf, and (5.10), we have

$$
\lim _{t \rightarrow \infty} \frac{\log |\log L(t)|}{\log t}=\frac{d}{d+\alpha}+\frac{\alpha}{d+\alpha} \lim _{t \rightarrow \infty} \frac{\log h(t)}{\log t}=1-\alpha \lim _{t \rightarrow \infty} \frac{\log x_{t}}{\log t} .
$$

An application of Lemma 5.3 completes the proof.

\section{Discussion And EXAmples}

We now discuss several specific classes of distributions $F_{q}$ to which our results apply directly. Recall the notation: $g(x)=\log \frac{1}{F_{q}\left(D_{0} / x\right)}, j(x)=x^{d+\alpha} g\left(x^{\alpha}\right), x_{t}=j^{-1}(t), h(t)=g\left(x_{t}^{\alpha}\right)$. For more clarity, our discussion will be divided into four subsections.

6.1. Distribution functions $F_{q}$ with an atom at zero. Suppose there exists $\kappa_{0}>0$ such that $F_{q}$ is continuous on $\left[0, \kappa_{0}\right]$ and $F_{q}(0)>0$. Then there are constants $C, \widetilde{C}>0$ such that

$$
-C \leqslant \liminf _{\lambda \searrow 0} \lambda^{d / \alpha} \log \ell(\lambda) \leqslant \limsup _{\lambda \searrow 0} \lambda^{d / \alpha} \log \ell(\lambda) \leqslant-\widetilde{C} \quad \text { and } \quad \lim _{\lambda \searrow 0} \frac{\log |\log \ell(\lambda)|}{\log \lambda}=-\frac{d}{\alpha} .
$$

Note that in this case we simply have $g(x) \asymp 1$ and $j(x) \asymp x^{d+\alpha}$ for large $x$, and therefore

$$
x_{t}=j^{-1}(t) \asymp t^{\frac{1}{d+\alpha}} \quad \text { and } \quad h(t) \asymp 1, \quad t \rightarrow \infty .
$$

In [17] we used Sznitman's coarse-graining method (the 'enlargement of obstacles method') to derive the Lifschitz tail in this case - for alloy-type potentials with random variables $q_{\mathbf{i}}$ having an atom at 0 . The paper was concerned primarily with $\Phi(\lambda)=\lambda^{\alpha / 2}, \alpha \in(0,2]$ (i.e. with the fractional powers of the Laplace operator and the Laplace operator itself) - in this case we were

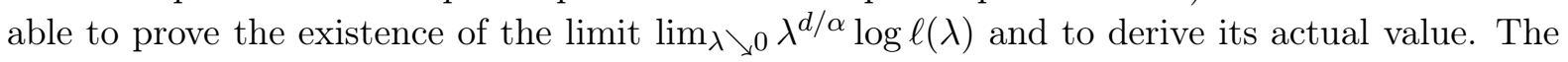
value of this limit was coherent with that obtained for Poisson-type potentials in [32, 38]. The method of [17] is also suitable to cover the case of some other subordinate processes, but with no precise scaling of principal Dirichlet eigenvalues at hand, in general we would be able to obtain only the statements for the lim sup and liminf, exactly as in (6.1) (cf. [16]).

6.2. Distribution functions $F_{q}$ with polynomial decay at zero. This section consists of two parts.

(1) Suppose that there exist $\gamma_{1}, \gamma_{2}>0, \kappa_{0}>0$ and constants $B_{1}, B_{2}>0$ such that

$$
B_{1} \kappa^{\gamma_{1}} \leqslant F_{q}(\kappa) \leqslant B_{2} \kappa^{\gamma_{2}}, \quad \kappa \in\left[0, \kappa_{0}\right] .
$$

This example covers all absolutely continuous distributions whose densities near zero behave polynomially or explode at most logarithmically fast (e.g. uniform, exponential, one-side normal, Weibull, arcsin, and many other distributions). In this case,

We then have

$$
g(x)=\log \frac{1}{F_{q}\left(D_{0} / x\right)} \asymp \log x, \quad \text { for large } x .
$$

$$
j(x)=x^{d+\alpha} g\left(x^{\alpha}\right) \asymp x^{d+\alpha} \log x, \quad x \rightarrow \infty,
$$

giving

$$
x_{t}=j^{-1}(t) \asymp\left(\frac{t}{\log t}\right)^{\frac{1}{d+\alpha}}, \quad t \rightarrow \infty
$$


and

i.e. for some constants $C, \widetilde{C}>0$

$$
h(t)=g\left(x_{t}^{\alpha}\right) \asymp \frac{\alpha}{d+\alpha} \log t, \quad t \rightarrow \infty
$$

$$
-C \leqslant \liminf _{t \rightarrow \infty} \frac{\log L(t)}{t^{\frac{d}{d+\alpha}}(\log t)^{\frac{\alpha}{d+\alpha}}} \leqslant \limsup _{t \rightarrow \infty} \frac{\log L(t)}{t^{\frac{d}{d+\alpha}}(\log t)^{\frac{\alpha}{d+\alpha}}} \leqslant-\widetilde{C} .
$$

Finally, for certain constants $C, \widetilde{C}>0$,

$$
-C \leqslant \liminf _{\lambda \searrow 0} \frac{\lambda^{d / \alpha}}{\log \lambda} \log \ell(\lambda) \leqslant \limsup _{\lambda \searrow 0} \frac{\lambda^{d / \alpha}}{\log \lambda} \log \ell(\lambda) \leqslant-\widetilde{C} \quad \text { and } \quad \lim _{\lambda \searrow 0} \frac{\log |\log \ell(\lambda)|}{\log \lambda}=-\frac{d}{\alpha} .
$$

(2) The asymptotics of the IDS in the loglog regime has been previously established by Kirsch ans Simon in 21] for random Schrödinger operators $-\Delta+\sum_{\mathbf{i} \in \mathbb{Z}^{d}} q_{\mathbf{i}}(\omega) W(x-\mathbf{i})$ with bounded random variables $q_{\mathbf{i}}$ satisfying the one-sided bound $B_{1} \kappa^{\gamma_{1}} \leqslant F_{q}(\kappa)$, under somewhat different assumptions on the single-site potential $W$. Observe that such a one-sided bound is not sufficient for determining the term $h(t)$ needed in the 'log' regime, even asymptotically: for example, when there is an atom at zero (cf. Section 6.1 above), then the one-sided bound holds, but $h(t) \asymp 1$, $t \rightarrow \infty$, while still $F_{q}(\kappa) \geqslant \mathbb{Q}[q=0] \geqslant B_{1} \kappa^{\gamma_{1}}, \kappa \leqslant \kappa_{0}$ - which should be contrasted with the results from part (1) above.

Our present approach generalizes the results of Kirch and Simon: we are able to derive the 'loglog statements' for both the integrated density of states and its Laplace transform from the more delicate statements in the 'log' regime. Indeed, as in this case there is a constant $c>0$ such that

it follows that

$$
g(x)=\log \frac{1}{F_{q}\left(D_{0} / x\right)} \leqslant c \log x, \quad \text { for large } x,
$$

and from Corollary 5.4 and Lemma 5.3 we get

$$
\lim _{x \rightarrow \infty} \frac{\log g(x)}{\log x}=0
$$

$$
\lim _{\lambda \searrow 0} \frac{\log |\log \ell(\lambda)|}{\log \lambda}=-\frac{d}{\alpha} \quad \text { and } \quad \lim _{t \rightarrow \infty} \frac{\log |\log L(t)|}{\log t}=\frac{d}{d+\alpha} .
$$

Note also that the result for the Laplace transform is new.

6.3. Distribution functions $F_{q}$ with exponential decay at zero. We now give an example what can happen when the decay of $F_{q}$ near zero is faster than polynomial. For a fixed $\gamma>0$ we let

$$
F_{q}(\kappa)=\mathrm{e}^{-\frac{1}{\kappa^{\gamma}}}, \quad \kappa>0
$$

We verify that in this case

$$
g(x)=\left(x / D_{0}\right)^{\gamma}
$$

and further

$$
j(x)=D_{0}^{-\gamma} x^{d+\alpha(1+\gamma)} \text { and } x_{t}=\left(D_{0}^{\gamma} t\right)^{\frac{1}{d+\alpha(1+\gamma)}},
$$

which gives

$$
h(t)=c t^{\frac{\alpha \gamma}{d+\alpha(1+\gamma)}}=c t^{1-\frac{d+\alpha}{d+\alpha(1+\gamma)}} .
$$

Consequently, the rate of decay for the Laplace transform of the IDS is

$$
t^{\frac{d}{d+\alpha}}(h(t))^{\frac{\alpha}{d+\alpha}}=t^{1-\frac{\alpha}{d+\alpha(1+\gamma)}}
$$

and there exist two constants $C, \widetilde{C}>0$ for which

$$
-C \leqslant \liminf _{\lambda \searrow 0} \lambda^{\frac{d}{\alpha}+\gamma} \log \ell(\lambda) \leqslant \limsup _{\lambda \searrow 0} \lambda^{\frac{d}{\alpha}+\gamma} \log \ell(\lambda) \leqslant-\widetilde{C} .
$$


The loglog limit is

$$
\lim _{\lambda \searrow 0} \frac{\log |\log \ell(\lambda)|}{\log \lambda}=-\frac{d}{\alpha}-\gamma
$$

It means that we observe an increase in the power of the exponent which is due to the fast decay of the cumulative distribution function of $q$. It should be noted that when $\gamma \rightarrow \infty$, then the rate of decay of the Laplace transform approaches $t$, which is the upper bound for the rate possible.

6.4. Distribution functions $F_{q}$ with double-exponential decay near zero. From (3.5) we see that $h(t) / t=x_{t}^{-d-\alpha} \rightarrow 0$ as $t \rightarrow \infty$, therefore the rate

$$
t^{\frac{d}{d+\alpha}}(h(t))^{\frac{\alpha}{d+\alpha}} \quad \text { with } \quad h(t) \asymp t, \quad t \rightarrow \infty,
$$

is never possible. However, it can happen that $\frac{\log h(t)}{\log t} \rightarrow 1$ as $t \rightarrow \infty$, which is illustrated by this example.

For the distribution whose CDF is given by

$$
F_{q}[\kappa]=\mathrm{e}^{1-\mathrm{e}^{\frac{1}{\kappa}}}, \quad \kappa>0
$$

we have

$$
g(x)=-\log F_{q}\left(D_{0} / x\right)=\mathrm{e}^{\frac{x}{D_{0}}}-1 .
$$

It then follows from Theorem 1.1 that there exist constants $C, \widetilde{C}, D, \widetilde{D}>0$ such that

$$
-C \leqslant \liminf _{\lambda \searrow 0} \lambda^{d / \alpha} \mathrm{e}^{-D / \lambda} \log \ell(\lambda) \quad \text { and } \quad \limsup _{\lambda \searrow 0} \lambda^{d / \alpha} \mathrm{e}^{-\widetilde{D} / \lambda} \log \ell(\lambda) \leqslant-\widetilde{C} .
$$

Consequently, we do not have the usual Lifschitz tail; the rate of decay of $\ell(\lambda)$ to zero is double exponential: we see that

$$
\widetilde{D} \leqslant \liminf _{\lambda \searrow 0} \lambda \log |\log \ell(\lambda)| \leqslant \limsup _{\lambda \searrow 0} \lambda \log |\log \ell(\lambda)| \leqslant D .
$$

This justifies the name: super-Lifchitz tail.

To determine the asymptotical rate for the Laplace transform $L(t)$ first observe that the function

$$
k(t):=\left(D_{0} \log \left(\frac{t}{\left(D_{0} \log t\right)^{(d+\alpha) / \alpha}}+1\right)\right)^{1 / \alpha}
$$

is the asymptotic inverse of the function $j(x)=x^{d+\alpha} g\left(x^{\alpha}\right)=x^{d+\alpha}\left(\mathrm{e}^{\frac{x^{\alpha}}{D_{0}}}-1\right)$ as $x \rightarrow+\infty$. Therefore,

$$
x_{t} \asymp k(t) \quad \text { and } \quad h(t) \asymp \frac{t}{(\log t)^{\frac{d}{\alpha}+1}}, \quad t \rightarrow \infty,
$$

resulting in the asymptotics

$$
-C \leqslant \liminf _{t \rightarrow \infty} \frac{\log L(t)}{t / \log t} \leqslant \limsup _{t \rightarrow \infty} \frac{\log L(t)}{t / \log t} \leqslant-\widetilde{C}
$$

and

$$
\lim _{t \rightarrow \infty} \frac{\log |\log L(t)|}{\log t}=1 .
$$

As the last remark observe that the last 'log log assertion' also follows directly from Corollary 5.4. without the prior knowledge of the asymptotic behavior of the functions $x_{t}$ and $h(t)$. 


\section{REFERENCES}

[1] M. Benderskii, L. Pastur: On the spectrum of the one-dimensional Schrödinger equation with random potential, Mat. Sb. 82 (1970) 245-256.

[2] K. Bogdan, T. Byczkowski: Potential theory for the $\alpha$-stable Schrödinger operator on bounded Lipschitz domains, Studia Math. 133 (1999) 53-92.

[3] J. Bourgain, C. E. Kenig: On localization in the continuous Anderson-Bernoulli model in higher dimension Invent. Math. 161 (2005), 389-426.

[4] R. Carmona, J. Lacroix: Spectral theory of random Schrödinger operators. Probability and its Applications. Birkhäuser, Boston, Inc., Boston, MA, 1990.

[5] R. Carmona, W.C. Masters, B. Simon: Relativistic Schrödinger operators: asymptotic behaviour of the eigenfunctions, J. Funct. Anal. 91, 1990, 117-142.

[6] L. Chaumont, G. Uribe Bravo: Markovian bridges: weak continuity and pathwise constructions, Ann. Probab. 39 (2), 2011, 609-647.

[7] Z.-Q. Chen, R. Song: Two-sided eigenvalue estimates for subordinate processes in domains, J. Funct. Anal. 226 (2005) 90-113.

[8] J. M. Combes, P. D. Hislop: Localization for some continuous, random Hamiltonians in d-dimensions. J. Funct. Anal. 124, 149-180 (1994).

[9] M. Demuth, J.A. van Casteren: Stochastic Spectral Theory for Self-adjoint Feller Operators. A Functional Analysis Approach. Birkhäuser, Basel 2000.

[10] R. Friedberg, J. Luttinger: Density of electronic energy levels in disordered systems, Phys. Rev. B 12 (1975) 4460-4474.

[11] M. Fukushima: On the spectral distribution of a disordered system and a range of a random walk, Osaka J. Math. 11, 1974, 73-85.

[12] M. Fukushima, H. Nagai, and S. Nakao: On an asymptotic property of spectra of a random difference operator, Proc. Japan Acad. 51 (1975) 100-102.

[13] F. Germinet, P. D. Hislop, A. Klein: Localization for Schrödinger operators with Poisson random potential. J. Eur. Math. Soc. (JEMS) 9 (2007), no. 3, 577-607.

[14] N. Jacob: Pseudo-Differential Operators and Markov Processes: Markov Processes and Applications. Vol. I, II, III. Imperial College Press, London 2001-2005.

[15] K. Kaleta, K. Pietruska-Pałuba: Integrated density of states for Poisson-Schrödinger perturbations of subordinate Brownian motions on the Sierpiński gasket, Stoch. Proc. Appl. 125 (2015), 1244-1281.

[16] K. Kaleta, K. Pietruska-Pałuba: Lifschitz singularity for subordinate Brownian motions in presence of the Poissonian potential on the Sierpiński gasket, Stoch. Proc. Appl. 128 (2018), 3897-3939.

[17] K. Kaleta, K. Pietruska-Pałuba: Lifschitz tail for alloy-type models driven by the fractional Laplacian, preprint 2019, available at arXiv:1906.03419

[18] K. Kaleta, R.L. Schilling: Progressive intrinsic ultracontractivity and heat kernel estimates for non-local Schrödinger operators, preprint 2019, available at arXiv:1903.12004

[19] W. Kirsch, F. Martinelli: On the density of states of Schrödinger operators with a random potential, J. Phys. A 15 (1982) 2139-2156.

[20] W. Kirsch, F. Martinelli: Large deviations and Lifshitz singularity of the integrated density of states of random Hamiltonians, Commun. Math. Phys. 89 (1983) 27-40.

[21] W. Kirsch, B. Simon: Lifshitz Tails for Periodic Plus Random Potentials, J. Stat. Phys. 42:5/6 (1986) 799-808.

[22] W. Kirsch, I. Veselić: Lifshitz Tails for a Class of Schrödinger Operators with Random Breather-Type Potential, Lett. Math. Phys. 94 (2010) 27-39.

[23] F. Klopp: Weak Disorder Localization and Lifshitz Tails, Commun. Math. Phys. 232 (2002) 125-155.

[24] F. Klopp: Weak Disorder Localization and Lifshitz Tails: Continuous Hamiltonians, Ann. Henri Poincaré 3 (2002) 711-737.

[25] I.M. Lifshitz, Energy spectrum structure and quantum states of disordered condensed systems, Soviet Physics Uspekhi 7 (1965), 549-573.

[26] J.M. Luttinger: New variational method with applications to disordered systems, Phys. Rev. Lett. 37 (1976) 609-612.

[27] G. Mezincescu: Bounds on the integrated density of electronic states for disordered Hamiltonians, Phys. Rev. B 32 (1985) 6272-6277.

[28] H. Nagai: On an exponential character of the spectral distribution function of a random difference operator, Osaka J. Math. 14 (1977) 111-116.

[29] S. Nakao: On the spestral distribution of the Schrödinger operator with random potential, Japan J. Math. Vol. 3 (1977), 111-139.

[30] L.A. Pastur: The behavior of certain Wiener integrals as $t \rightarrow \infty$ and the density of states of Schrödinger equations with random potential, (Russian) Teoret. Mat. Fiz. 32 (1977) 88-95.

[31] K. Pietruska-Pałuba: The Lifschitz singularity for the density of states on the Sierpinski gasket, Probab. Theory Related Fields 89 (1991), no. 1, 1-33. 
[32] H. Okura: On the spectral distributions of certain integro-differential operators with random potential, Osaka J. Math. 16 (1979), no. 3, 633-666.

[33] M. Reed, B. Simon: Methods on Modern Mathematical Physics. Vol. 4: Analysis of Operators, Academic Press, 1978.

[34] M. Romerio, W. Wreszinski: On the Lifshitz singularity and the tailing in the density of states for random lattice systems, J. Stat. Phys. 21 (1979) 169-179.

[35] R. Schilling, R. Song, Z. Vondraček: Bernstein Functions, Walter de Gruyter, 2010.

[36] K. Sato: Lévy Processes and Infinitely Divisible Distributions. Cambridge University Press, Cambridge 1999.

[37] B. Simon: Lifshitz tails for the Anderson model, J. Stat. Phys. 38 (1985) 65-76.

[38] A.S. Sznitman: Lifschitz tail and Wiener sausage I, J. Funct. Anal. 94 (1990), 223-246.

(K. Kaleta) Faculty of Pure and Applied Mathematics, WrocŁaw University of Science and Technology, ul. Wybrzeże Wyspiańskiego 27, 50-370 Wroceaw, Poland

E-mail address: kamil.kaleta@pwr.edu.pl

(K. Pietruska-Pałuba) Institute of Mathematics, University of WarsaW, ul. Banacha 2, 02-097 Warszawa, Poland

E-mail address: kpp@mimuw.edu.pl 